\title{
ATSC 3.0 for Future Broadcasting: Features
}

\section{and Extensibility}

\author{
Sungjun Ahn, \\ Sunhyoung Kwon, \\ Seok-Ki Ahn, \\ Hoiyoon Jung and \\ Sung-Ik Park
}




\title{
ATSC 3.0 for Future Broadcasting: Features and Extensibility
}

\author{
Sungjun Ahn, Sunhyoung Kwon, Seok-Ki Ahn, Hoiyoon Jung, and Sung-Ik Park
}

\begin{abstract}
A recent development of Advanced Television Systems Committee (ATSC) 3.0 has made over-the-air services of a $4 \mathrm{~K}$ ultra-high-definition and a simultaneous multiple highdefinition soft-landed to reality. However, ATSC 3.0 is in essence designed to be forward compatible, and is hence able to provide better extensible features to enrich the next media era beyond this initial deployment. This paper introduces several selected features of ATSC 3.0 that could play a prominent role in the near future. This investigation encompasses the optional technologies defined in ATSC 3.0 physical-layer which can improve single frequency network integrity, enable additional features in other domains, or further widen the throughput capability. Majorly focused on the extensibility of ATSC 3.0, we also elaborate on possible inter-network cooperation with broadband and cellular systems.
\end{abstract}

Index Terms-ATSC 3.0, physical-layer, digital terrestrial broadcasting, broadcast-broadband convergence

\section{INTRODUCTION}

$\mathrm{O}$ NE of the most notable evolution in modern terrestrial broadcasting is a development of new digital terrestrial television (DTT) standard, so-called Advanced Television Systems Committee (ATSC) 3.0 [1]. To satisfy a wide variety of requirements for the upcoming media era, this new standard has been designed without considering backward compatibility to the legacy ATSC networks [2]. From the first Call for Proposals (CfP) in March 2013, the overall establishment had been progressed across the entire system layers until the first finalized release in January 2018. The first commercial launch of ATSC 3.0 has been held in the Republic of Korea since the mid of 2017 [3]. Driven by a group of major broadcasters, the United States has as well commenced ATSC 3.0 broadcasting since 2020. In the wake of the $600 \mathrm{MHz}$ spectrum repack recently concluded in the United States [4], ATSC 3.0 is planned to be distributed nationwide by the end of 2020. Currently, the on-air services in Korea are in the majority oriented to streaming a $4 \mathrm{~K}$ ultrahigh-definition (UHD) video, while the United States stakeholders focus on providing dozens of service channels over a single $6 \mathrm{MHz}$-width radio frequency $(\mathrm{RF})$ band, which may boost a cord-cutting faster.

Thanks to a manifold of cutting-edge physical-layer

This work was supported by Institute of Information \& communications Technology Planning \& Evaluation (IITP) grant funded by the Korea government (MSIT) (2020-0-00846, Development of Convergence Transmission Technology for 5G and ATSC 3.0 Networks). (Corresponding author: Sung-Ik Park.)

Sungjun Ahn, Sunhyoung Kwon, Seok-Ki Ahn, Hoiyoon Jung, and SungIk Park are with the Media Research Division, Electronics and technologies integrated therein, ATSC 3.0 ensures 30\% enhanced capacity over ATSC 1.0 for the same coverage [5]. More than this distinct throughput improvement, the new standard also provides an intensely flexible transmission unlike the previous DTT standards existed ever [6]-[8]. For example, even the combination of forward error correction (FEC) and constellation mapping alone has 360 different options. Up to $60 \mathrm{Mbps}^{1}$ over up to 64 different physicallayer pipes (PLPs) are capable within a single RF channel thereon [9]. A combination of various interleaving and waveform parameters are also given selectable by purpose, thereby allowing accurate customization for each use case and single frequency network (SFN) topology [10], [11]. Such physical-layer flexibility enables an SFN infrastructure to offer mobile and stationary services or the mixture thereof without undesirable leakage of resources, hence acquiring substantial network fidelity and resource/cost efficiency. Impressively, this physical-layer flexibility can potentially be extended wider by leveraging a future compatible versioning of bootstrap signals [12].

Another noticeable feature of ATSC 3.0 is an Internet Protocol (IP)-based design across the entire system [13]. The overall IP compatibility grants ATSC 3.0 an extensibility to cooperate with other radio or wired access networks [14], [15]. This possibility, so-called broadcast-broadband convergence, has brought enormous attention from the industry since it can create new service opportunities for which improves and enriches the experience [16]. Beyond the physical-layer flexibility within an ATSC 3.0 standalone network, such converged networks can open the door toward another dimension of flexibility upon system configuration [17]. As pointed out in various publications, the converged network can become better enhanced by being combined with functional gadgets in ATSC 3.0, such as a layered division multiplexing (LDM) [18], [19].

Focusing on the service extensibility of ATSC 3.0, this paper introduces several selected features of ATSC 3.0 that could play a prominent role beyond the initial deployment. This investigation encompasses the optional technologies defined in ATSC 3.0 physical-layer which can improve SFN integrity, enable additional features in other domains, or further widen the throughput capability. We also elaborate on possible inter-network cooperation with broadband and

Telecommunications Research Institute (ETRI), 218 Gajeong-ro, Yuseonggu, Daejeon, 305-700 S KOREA (e-mail: \{sjahn, shkwon, seokki.ahn, junghy,psi76\}@etri.re.kr)

${ }^{1}$ This maximum throughput is subjected to a single physical channel usage. Multiple-input multiple-output (MIMO) [20] or channel bonding (CB) [21] operations could double the capacity, as will be described below. 
cellular systems. We therein present a particular service example that realizes an interplay between the boundaries of broadcast and broadband planes. The discussions in this paper are expected to foster a progressive DTT environment that can readily evolve to support emerging media demands.

This paper is organized as follows. Section II introduces several forward-looking features of ATSC 3.0 physical-layer, and Section III discusses the extensibility of ATSC 3.0 mainly in a broadband convergence perspective. The verification test results for ATSC 3.0 system performance are summarized in Section IV, and Section V finally concludes the paper with some remarks.

\section{New Features of ATSC 3.0 PhySiCAL-LAyer}

ATSC 3.0 physical-layer in essence provides a broad range of improvement over throughput, reliability, flexibility, and future extensibility by using state-of-the-art bit-interleaved coded modulation (BICM) and orthogonal frequency division multiplexing (OFDM) framing technologies. Meanwhile, ATSC 3.0 offers more advanced options for service opportunities by supporting further distinguished features that have hardly been considered in previous DTT systems. This section describes several selected features which have been newly adopted in ATSC 3.0 system and made the new standard further promising.

\section{A. Layered Division Multiplexing (LDM)}

For decades, the idea of non-orthogonal multiplexing (NOM) has been probed to be a superseder of traditional time- and frequency-domain orthogonal multiplexing schemes [22]-[24]. The efforts have been mainly focused on the power-domain NOM, i.e., superposition coding, and have revealed its notable gain on spectral efficiency ${ }^{2}$. The first standardized form of NOM was the multi-user simultaneous transmission (MUST) ${ }^{3}$ in long-term evolution-advanced (LTE-A) [25]. However, due to some practical problems including a difficulty at uplink scheduling, MUST unfortunately has not yet been commercialized in the industry.

On the other hand, the $2^{\text {nd }}$ effort for standardizing NOM, LDM of ATSC 3.0, has attracted a significant attention to fit in the broadcasters' service plans well [11],[26]-[39]. Particularly the fundamental excellence of power-domain NOM in unequal error protection (UEP) scenarios has been highlighted [40]. Celebrated use cases multiplexing mobile, indoor HD, and fixed UHD services are generally referred to promote LDM, where different coverage or quality of service (QoS) is intended for each service therein ${ }^{4}$. Since broadcasters may attempt to serve as many programs as possible to various types of users within limited spectral resources (that they are subjected to), such spectral efficiency gain from LDM would be significantly desirable in a commercial sense. In addition, unlike MUST in the cellular access system, LDM for DTT is already viable in practice because broadcasting does not have any difficulties on user pairing and scheduling. To be mentioned, the 2018 Winter Olympic Games were broadcasted over ATSC 3.0 LDM in

A superposition coding has been decently proved to be optimal for broadcast channels, in an information-theoretic sense [41].

In specific words, MUST is a non-orthogonal multiple access (NOMA) scheme rather than a simple NOM.

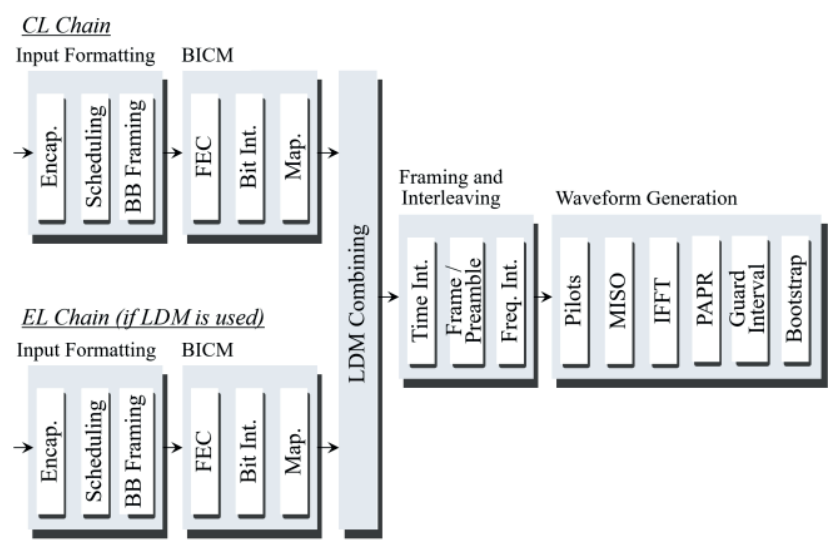

Fig. 1. Block diagram for ATSC 3.0 LDM transmission.

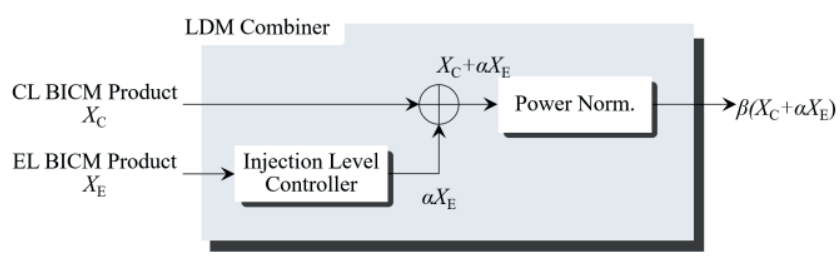

Fig. 2. Description of LDM combining block.

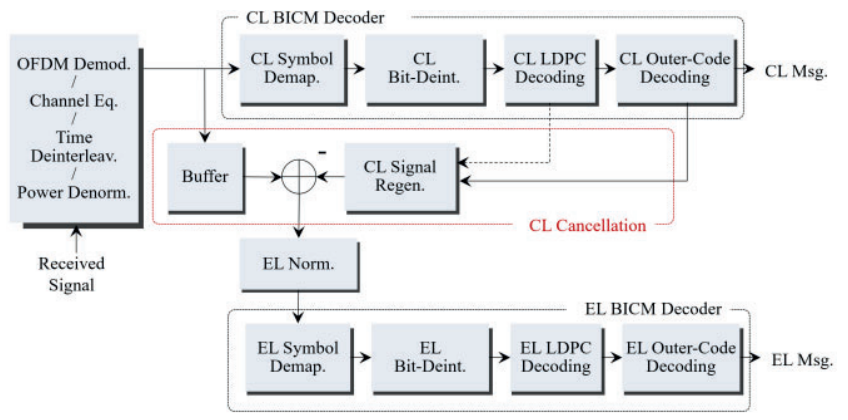

Fig. 3. Block diagram for ATSC 3.0 LDM decoding procedure.

Raleigh, United States. In this on-air demonstration, HD and $4 \mathrm{~K}$ UHD programs with different target coverages were transmitted on the same radio channel [42].

ATSC 3.0 LDM is specifically a two-layer NOM that combines each, differently powered, layer signal cell-by-cell. The unit cell herein refers to a single constellation-mapped signal component that occupies a single subcarrier part within an OFDM symbol. The signal layers of ATSC 3.0 LDM are specified into a core layer (CL) and an enhanced layer (EL), which denote the stronger and the weaker signal layers, respectively. The baseband power ratio between those layers is determined by a variable so-called an injection level, which is explicitly informed through L1-Detail signaling.

As shown in Fig. 1 and Fig. 2, the post-BICM products of two independent baseband streams are arithmetically combined in a weighted-sum manner. The overall constellation figure of the LDM-combined signal then becomes a form of which the EL constellation is duplicated for each point of the CL constellation. Note herein that every participating PLP undergoes the BICM process independently. The BICM configurations for CL and EL

\footnotetext{
${ }^{4}$ One convincing use case is a scalable video service over LDM, which views UHD to stationary user and moves into HD when the user goes through somewhat harsh environment, such as mobile reception.
} 


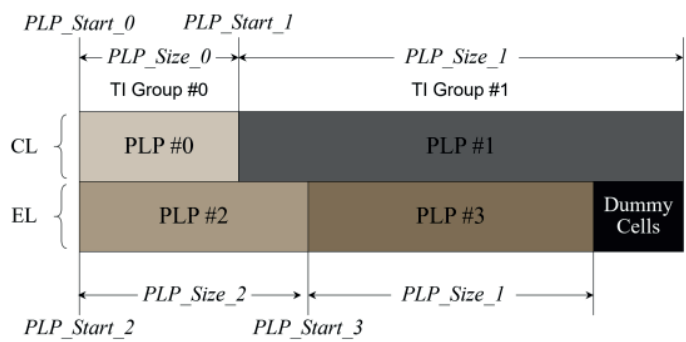

Fig.4. Example of cell positioning and TI under LDM configuration.

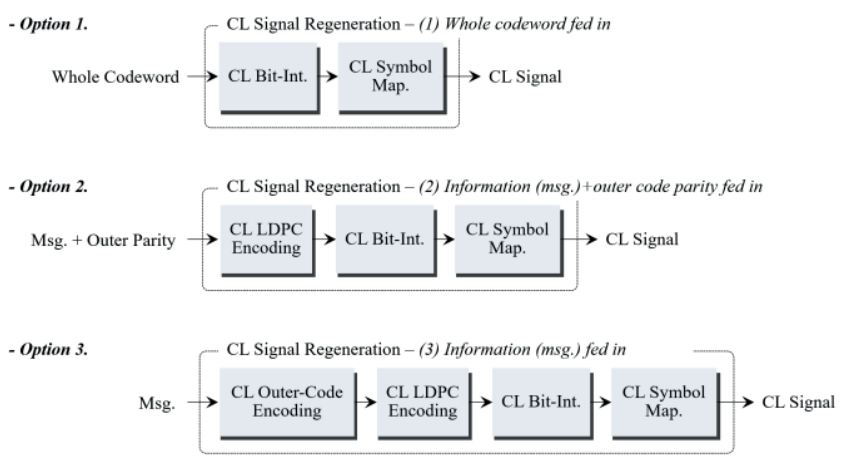

Fig. 5. Implementation options for CL regeneration within SIC block.

PLPs and the contents therein are therefore not subjected to each other. This independency empowers ATSC 3.0 LDM to be versatile to various service attempts, by flexibly satisfying each intended coverage and throughput per PLP.

To offer reasonable signal processing complexity, memory requirement, and end-to-end latency, the interleaving, framing, and waveform generation are applied after the layer combining [27]. That is, for instance, the CL and EL cells paired to each other go through exactly the same timeinterleaving (TI). This arrangement reduces the entailed signaling overhead also. The shared TI is defined within the signaling fields subjected to CL PLPs, and each EL cell is just led to follow the same sequence with the CL cell superposed to it. To be clarified, it is not necessary to align the boundaries of CL and EL PLPs to each other. The TI for each EL cell is determined by the cell position within a subframe, and particularly depends on which CL PLP is superposed on it. An auxiliary unit TI group is accordingly introduced to clarify TI coordination in complex scenarios. For the sake of clarity, convolutional TI over an LDM frame is carried out on a TI group basis as depicted in Fig. 4 [11].

Since the signal division of LDM does not reside in the time and frequency domain ${ }^{5}$, it is also possible to use LDM in combination with time- or frequency-division multiplexing (TDM/FDM). One possible example is a time-divided LDM (TLDM), whose TDM slices are LDM-combined PLPs. In a similar sense, layered-TDM (LTDM), frequency-divided $L D M$ (FLDM), and layered-FDM (LFDM) are available as well. Such combinatorial use further fleshes out the transmission flexibility, bringing more opportunity to enhance spectral efficiency. For a specific example transmitting immersive audio, mobile HD, and fixed UHD within a frame, [11] reported that LTDM could achieve 120\% capacity gain over TDM for the mobile HD PLP.

${ }^{5}$ Note that LDM does not reside in the same place with TDM and FDM even in a transmit chain perspective. TDM and FDM are carried out as a part of framing block, whereas LDM occurs earlier.
Although there is no stipulated restriction on BICM configuration for ATSC 3.0 LDM, it is typical to use a better robust modulation-and-coding (ModCod) combination for CL than that of EL. [8], the recommended guideline document for ATSC 3.0 physical-layer, further suggests to use up to 64 non-uniform constellation (NUC) for CL PLP, and to use up to 7/15-rate low-density parity check (LDPC) code if the CL PLP is modulated with 64 NUC.

Such considerations for CL robustness (relative to EL PLPs) attributes to inter-layer interference (ILI) due to the EL injection. In general, a typical ATSC 3.0 receiver attempts to retrieve the CL signal directly from the received signal but does not extract any constructive information from EL components during then. When a CL PLP is intended, EL signals are therefore regarded as inevitable ILI that degrades the signal quality. Sufficiently robust BICM configurations are hence considered for CL PLPs in order to make up such degradation. However, not to be confused, this does not imply that using LDM penalizes the spectral efficiency compared to TDM (and FDM). Though there is an implicit throughput penalty from ILI, LDM CL can instead exploit every data cell resource within a frame whereas only a part is available to the TDM counterpart.

On the contrary, EL decoding proceeds in an ILI-free condition, thanks to successive interference cancellation $(\mathrm{SIC})^{6}$. According to a typical ATSC 3.0 receiver architecture described in Fig. 5, the terminal restores CL signals first and then uses them to remove the corresponding component from the received signal prior to the EL decoding. The effective EL signal fed into the CL BICM decoder is thereby refined to have a substantially higher signal-to-interference plus noise ratio (SINR) than the ILI-included signal previously fed into CL BICM decoder. In this end, desirable reliability is ensured for ultra-high throughput transmissions over EL.

As can be noticed, LDM decoding involves some additional computations and buffering due to SIC. However, it has been verified that the entailed extra latency, memory and complexity lie in an acceptable, may be even negligible, range. Thanks to a careful design of ATSC 3.0 that allows CL and EL to share a common signal structure (e.g., TI and other OFDM/reference signal organizations), the additional burdens were practically demonstrated to be insignificant via official plug-in tests supervised by ATSC.

Depending on implementations, the receiver complexity with respect to SIC can be further reduced more. To this end, [27] and [8] have introduced two alternative SIC implementations that do not regenerate the CL codeword down from the raw data level. As described in [27], the lowcomplexity receiver can be implemented by directly extracting partially decoded codeword from the CL BICM decoder for canceling out the CL signal. Compared to the base strategy that uses the end product of CL BICM decoder, those alternative schemes omit decoding and re-encoding processes for FEC, and then significantly reduce the computation and latency. Such procedure-drops may bring the possibility of cross-layer interference (CLI) from CL to EL, i.e., the remainders after SIC. However, EL decoding is

${ }^{6}$ A superposition coding generally operates in a pair with SIC at the receiver-side. 
typically intended in a stationary environment with high signal-to-noise ratio (SNR), since the ModCods would be configured to have a sufficient SNR headroom between the CL and EL decoding. Therefore, quasi-error-free demapping of CL PLPs would probably be achieved, so that would get rid of the CLI problem in practice.

In order to assure a complete success of CL decoding as a premise to EL decoding instances, [8] recommends setting the injection level to be at least $3 \mathrm{~dB}$ greater than the nonLDM case threshold-of-visibility (ToV) of the least robust CL PLP. This additional headroom of $3 \mathrm{~dB}$ is heuristically determined in a rule-of-thumb sense, particularly to cover possible implementation losses.

\section{B. Transmitter Identification (TxID)}

Under a critical spectrum scarcity these days, DTT over an SFN has turned out significantly attractive due to its minimal spectrum usage. A coordinated transmission of SFN makes it possible to serve even a nation-wide territory with a single RF channel, but requires cautious deployment and orchestration among the transmitters.

For better accuracy of the SFN coordination, ATSC has introduced an idea to radiograph the impulse response from each transmitter, so that makes each transmitter's channel component distinguishable from the others. This idea, referred to as TxID, is achieved by inserting additive radio watermark signals (so-called TxID signals) into the transmit signals [43]-[52]. A code orthogonality principle of codedivision multiplexing (CDM) underlies this feature. When the TxID signals are uniquely assigned to transmitters, it becomes able to figure out the contribution (and interference as well) of each transmitter's emission to the received signal at once. It is an impressive benefit that this does not require any hassle of isolating the signal from each transmitter, i.e., turning the others off. Worthwhile to mention in addition, TxID signals are transparent to the legacy ATSC 3.0 receiver. Truly being a watermark, the TxID signals are seen as background noise for decoding the message-bearing frames.

ATSC 3.0 uses a set of binary phase-shift keying (BPSK)modulated Gold code sequences for TxID [48]. The length of a single sequence block therein is $8191\left(=2^{13}-1\right)$ samples, while the TxID signal in a single frame can consist of multiple repetition of such a unit sequence. Let us henceforth denote those unit sequences as TxID sequences. Based on the CDM capability of TxID sequences, up to $8192\left(=2^{13}\right)$ different transmitters can be uniquely indicated at the same time.

After being BPSK-modulated, the TxID signal is arithmetically combined with the baseband waveform of the $1^{\text {st }}$ preamble symbol, sample-by-sample. To avoid possible data contamination that TxID signal injection can bring out, ATSC 3.0 restricted the TxID signals to be injected only in the preamble period, whose FEC protection is typically given stronger than payloads. Even so, a broadcaster should pay particularly careful attention to determining the TxID injection level (i.e., the relative power level of TxID signal compared to the preamble transmission) to balance well between the performances of service acquisition (i.e., preamble decoding) and TxID signal detection.

As depicted in Fig. 6, the beginning of every TxID signal is precisely aligned with the $1^{\text {st }}$ sample part of the preamble
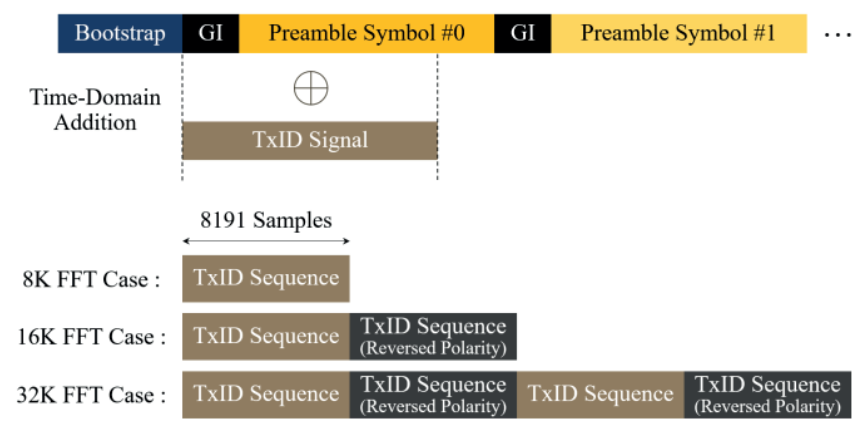

Fig. 6. TxID signal injection for various FFT sizes

interval (guard interval included). Once mentioned above, an 8191 samples-length TxID sequence can be repeated multiple times, thereby allowing the TxID signal to be better detectable. While then, the TxID signal should coexist only with the $1^{\text {st }}$ preamble symbol. The number of repetitions is therefore determined by the size of fast Fourier transform (FFT) applied to preamble signals.

In case an $8 \mathrm{~K}$ FFT is applied to preambles, for example, a TxID sequence is injected once per frame. Note herein that the duration of the $1^{\text {st }} 8 \mathrm{~K}$ FFT-modulated preamble symbol can contain up to one single TxID sequence. On the other hand, the $1^{\text {st }}$ preamble symbol's duration is doubled if $16 \mathrm{~K}$ FFT is used. In this $16 \mathrm{~K}$ FFT case, the TxID sequence is duplicated into two sequence blocks and then consecutively transmitted. The $2^{\text {nd }}$ sequence block therein is arranged to have the opposite polarity to the $1^{\text {st }}$ TxID sequence block, specifically to remove out the direct current component. As for $32 \mathrm{~K}$ FFT configurations, a TxID sequence block is repeated four times. Likewise, the $2^{\text {nd }}$ and $4^{\text {th }}$ TxID sequence blocks are modulated to have the opposite polarity to that of the $1^{\text {st }}$ and $3^{\text {rd }}$ blocks, while the $3^{\text {rd }}$ block is just a repetition of the $1^{\text {st }}$ block. Such topologies are briefly visualized in Fig. 6 .

The TxID signals can be detected by means of auto- and cross-correlations, and tend to be detected better when they are injected with higher power level [49]-[52]. However, excessively high TxID injection levels could rather disturb decoding preamble signals, and therefore should be avoided. Even more, a regulation issue restricts the TxID injection level as well. Since transmitters do not normalize the power of baseband signals after combining TxID signals, highpowered TxID signals could increase the net transmit power much. In effect, the transmit power is in practice strictly regulated by governments. Generally speaking, it is not allowed to go beyond the granted transmit power by more than its 5\%. The TxID injection level should hence be lower than $-15 \mathrm{~dB}$. In this regard, TxID injection levels from $-45 \mathrm{~dB}$ to $-15 \mathrm{~dB}$ are recommended to comply with the regulation.

In fact, TxID signals may not be detected that clearly for some instances in the real field. For such tough circumstances, the receiver can consider averaging the received TxID signals over a plurality of physical-layer frames. This method, socalled ensemble averaging, can reduce the effective noise seen in the received TxID signals, especially when non-TxID signal components are uncorrelated across frames. Back in the days with ATSC 1.0 systems, data frames (excluding pilot tones) were barely correlated with each other, and ensemble averaging could thereby achieve its maximum ability [43][47]. However, on the contrary, the preamble blocks of ATSC 
3.0 are pretty much correlated among frames, specifically for pilot elements scattered therein. Precisely, according to [51], the $1^{\text {st }}$ preamble symbol has turned out to have $52.58 \%$ $76.65 \%$ randomness. For this reason, ensemble averaging for ATSC 3.0 cannot improve the visibility of TxID signals by more than $4 \mathrm{~dB}$, whereas it could do significantly better in ATSC 1.0 systems.

To overcome such a limited ability for detecting the ATSC 3.0 TxID signal, [49]-[52] brought an idea of interference canceling into TxID detection. The idea is to remove preamble signal components from the received signal before feeding it into the correlation procedure. In particular, [51] proposed three types of detection methods: Pilot cancellation; whole preamble cancellation (WPC) with full LDPC decoding; and hard decision-based WPC. Those methodological branches arose from the tradeoff between detection performance and computational complexity. For example, the pilot cancellation method requires the least computation but is feasible only when ensemble averaging is executed over many frames. On the contrary, the WPC based on thorough decoding enhances TxID detection the best. Still, this exhaustive method involves much more computation which LDPC decoding brings with. The last option came out from this context, as a simplified version of WPC. This simplified technique regenerates preamble cancellation signals back from hard decision demapping over the preamble cells, which are modulated by quadrature phaseshift keying (QPSK), instead of performing complete decoding for FEC codewords. This simplified WPC lies between the previous two methods in terms of the performance-complexity tradeoff, providing acceptable detection performance and reasonable complexity.

Did not remain only as a concept, the aid of preamble cancellation has also been implemented in real hardware TxID detectors [51]. According to the laboratory and field test results in [51], WPC could assist the TxID detector to detect the TxID signals injected even $15 \mathrm{~dB}$ lower than what the conventional, correlation-only, method can detect. This enhanced detection capability allows the ATSC 3.0 broadcasters to reduce the injection power of TxID signals, so that relieves interference for decoding preambles. The network-scale gain of preamble cancellation was also theoretically proved in [50].

To be emphasized, the field experiments were done in the actual commercial network as well. Note again that the public ATSC 3.0 services in South Korea are currently on-air. The SFNs for those services include abundant transmitters in them, which are deployed over wide geographical areas. To manage such a complex network topology, TxID is often employed in the major broadcaster's on-air transmission in reality. The study [53], for example, presented the field measurement results that were drawn from a public ATSC 3.0 SFN in the Seoul metropolitan area. There, in [53], some snapshots of TxID-analyzed channel profiles were provided to show the real environment of dense SFN in the Seoul metropolitan.

\section{MIMO and Channel Bonding (CB) for Higher Data Rate Transmission}

In another vein, there also has been an effort to extend the pipe-width of ATSC 3.0. Although multiple videos with $4 \mathrm{~K}$

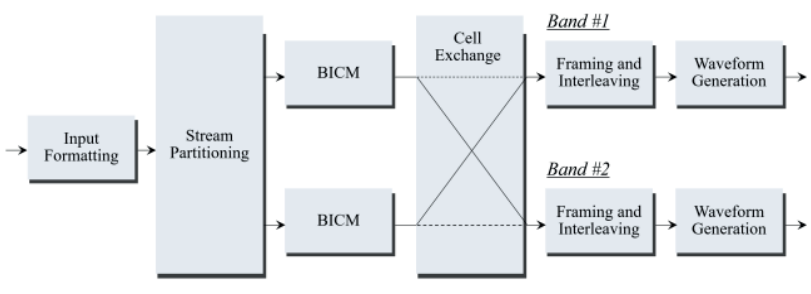

Fig. 7. ATSC 3.0 CB transmitter structure.

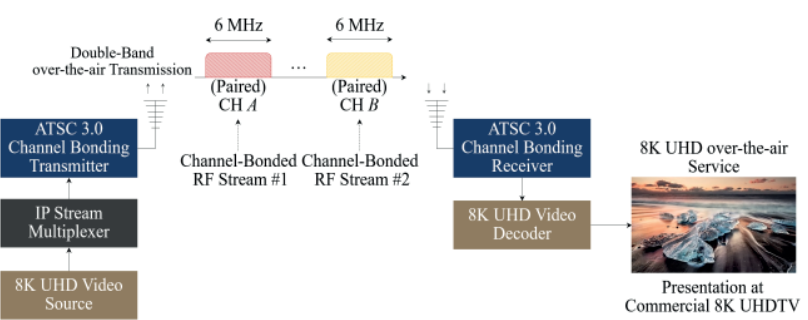

Fig. 8. Block diagram of ATSC 3.0 CB system.

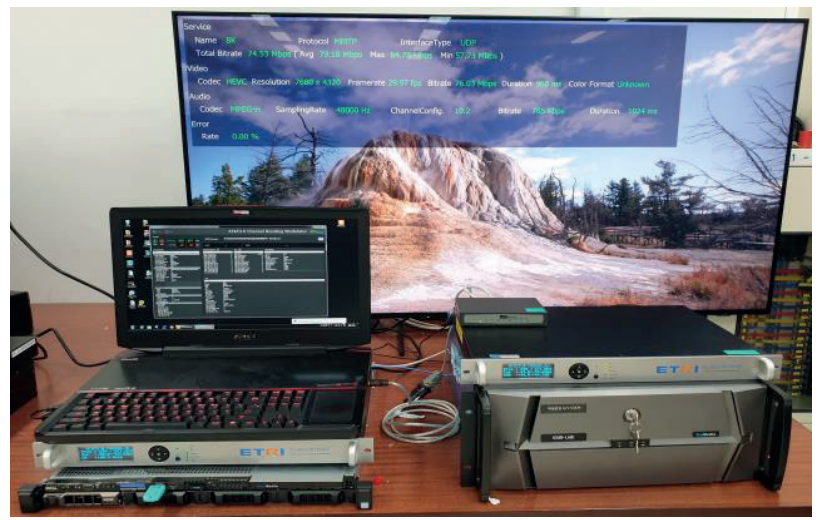

Fig. 9. 8K UHD system based on ATSC 3.0 CB.

resolution were already viable in baseline system configurations, ATSC 3.0 additionally defined two optional technologies for increased capacity: MIMO and CB [20], [21]. The intent behind those optional systems was to support future plans beyond $4 \mathrm{~K}$ UHD, such as $8 \mathrm{~K}$ videos and virtual reality, by doubling the pipelines. In specific, ATSC 3.0 MIMO transmissions exploit two polarization channels by employing two antennas each for the transmitter and receiver. On the other hand, CB technology utilizes two RF channels for increasing system capacity.

As for the CB in ATSC 3.0, two types of CB transmission are defined: A plain $\mathrm{CB}$ and a $\mathrm{CB}$ with SNR averaging. The plain $\mathrm{CB}$ transmits the data streams separately for each RF channel by configuring them independently, instead of applying the same system parameter set. Otherwise, if SNR averaging is applied to $\mathrm{CB}$, data cells are exchanged between two paired radio channels to obtain frequency diversity. However, transmissions over both radio channels shall share the same system parameters in this case [7].

Fig. 7 describes the procedure of ATSC 3.0 CB. The stream partitioning block first splits the input stream into two streams, and each stream is delivered to a different BICM block. The cell exchange block is enabled if SNR averaging is used, but remains disabled for the plain $\mathrm{CB}$. If enabled, the cell exchange block selectively exchanges the even-index data cells between the data stream paths. That is, the cell exchange is executed once for every two data cells, and hence the oddindex cells remain in the same path. This cell exchange 


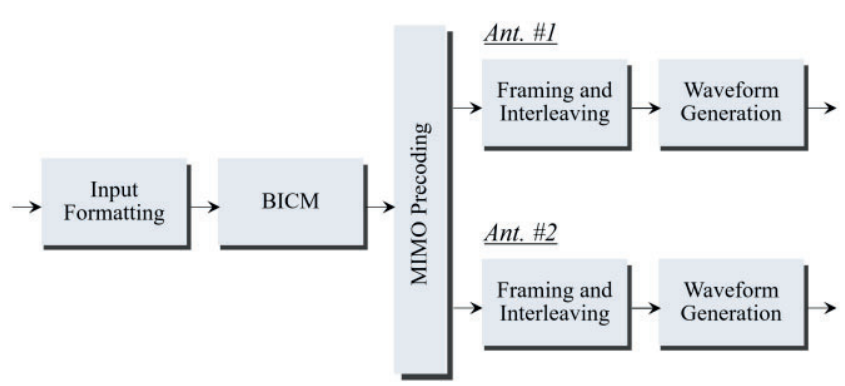

Fig. 10. ATSC 3.0 MIMO transmitter structure

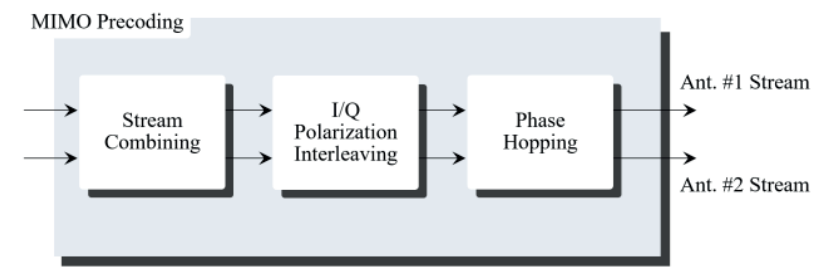

Fig. 11. ATSC 3.0 MIMO precoding structure

between separated channels disperses the frequency-selective fading effect, and as a result, acquires frequency diversity in an overall sense [54], [55]. To be noted, every CB transmission in ATSC 3.0 does not require the intended RF channels to be adjacent to each other.

An end-to-end system for ATSC 3.0 CB was implemented in reality as well, particularly to demonstrate a delivery of $8 \mathrm{~K}$ UHD or multiple 4 K UHD services over CB [56]. Fig. 8 briefly describes the $8 \mathrm{~K}$-over-CB system presented in [56]. An 8K UHD video, encoded by High Efficiency Video Codec (HEVC), was first IP-encapsulated and then fed into the ATSC 3.0 CB transmitter. The CB transmit signals, which went through the dedicated modulation chains shown in Fig. 7, were transmitted over two different wireless bands. Again, those RF bands could be either contiguous or non-contiguous. On the counterpart, the ATSC 3.0 CB receiver demodulated the received dual-channel signals to produce a combined output. The $8 \mathrm{~K}$ video decoder then retrieved the video source from this output, and finally presented $8 \mathrm{~K}$ imageries at the commercial 8K UHD television (UHDTV) display.

The implementation in [56] was in practice found to serve 8K UHD program reliably over two $6 \mathrm{MHz}$ wireless channels Fig. 9 shows an instance of laboratory trials. Particularly, transmissions of an $80 \mathrm{Mbps}$ bit-rate video was demonstrated in those trials. At the time of this writing, the implemented CB system was examined to be capable of transceiving a 110 Mbps video seamlessly in laboratory environments.

In another vein, ATSC 3.0 also supports a cross-polarized MIMO technology for increased capacity [57]. This technique utilizes only one single RF channel band but requires a pair of horizontally and vertically polarized antennas to be installed at each transmitter and receiver [58]. Fig. 10 depicts the transmitter architecture of ATSC 3.0 MIMO. It reuses most of the system blocks from the singleantenna operation, but MIMO mapping (MAP) ${ }^{7}$ and MIMO precoding blocks are newly introduced.

ATSC 3.0 MIMO carries out three types of precoding for transmission: Stream combining, in-phase/quadrature-phase

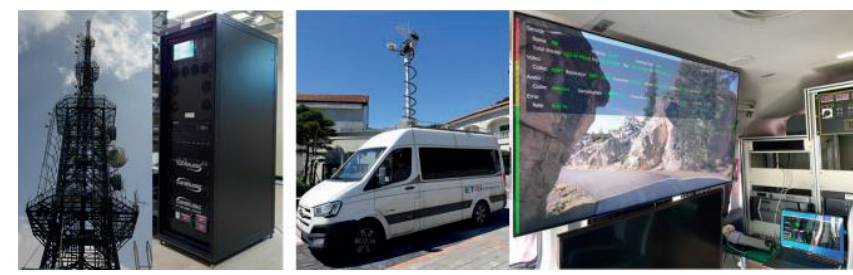

Fig. 12. ATSC 3.0 MIMO field experiments - Facilities and reception status.

(I/Q) polarization interleaving, and phase hopping (see Fig.11.). Stream combining reprojects a pair of the demultiplexed constellation symbols by applying a rotation matrix. The rotation angle is determined according to ModCod configuration. I/Q polarization interleaving then switches the imaginary part of the symbol between two data paths. In subsequence, the phase-hopping rotates the phase of the second data path's symbol. By means of those precoding procedures, MIMO transmissions can be better protected from imbalanced fading in an interleaving manner.

An end-to-end MIMO system fully compliant with ATSC 3.0 physical-layer standard has been implemented as well. The real environment performance of the implemented system has been examined by field testing, as shown in Fig. 12. Seamless delivery of $110 \mathrm{Mbps}$ data has been confirmed in the laboratory, and 105 Mbps delivery has been exhibited through field trials.

\section{EXTENSIBILITY OF ATSC 3.0}

One prominent feature that allows ATSC 3.0 to be distinct from previous standards is extensibility. This extensibility mainly owes to the overall IP-based design of ATSC 3.0, so that enables efficient cooperation with other non-ATSC 3.0 networks, such as a broadband cellular. This section summarizes recent evolutions on ATSC 3.0 extensibility, particularly focusing on the convergence with up-to-date broadband networks.

\section{A. Convergence with Broadband}

A part of the underlying philosophy on the design of ATSC 3.0 is to make an extensible system finding itself compatible with its future versions, or to the interplays with other networks' interfaces. A definition of bootstrap versioning and all-IP-based system design is the result of such principle. This consideration of extensibility lies in the same vein to the global trend in network designs: To exemplify thereof, the $5^{\text {th }}$ generation wireless standard $(5 \mathrm{G})$ can first be referred, which pursues establishing a macro topology to interact with various heterogeneous (including non-5G) networks. Since the user environments and service purposes are coming highly diverged, such an inter-network collaboration is foreseen to have an influential edge to optimize deliveries and broaden the system capability.

The recent interest of the media and entertainment (M\&E) industry has been particularly on the conjunction between ATSC 3.0 and broadband. Such an idea, popularly called as a broadcast-broadband convergence ( $B C B b C)$, has been seen as an opportunity for DTT to grab user interactivity at hand [16]. As one may come up with the first, this interactivity can

The MIMO MAP block consists of MIMO demultiplexing and constellation mapping sub-blocks, and is included in the BICM block. 


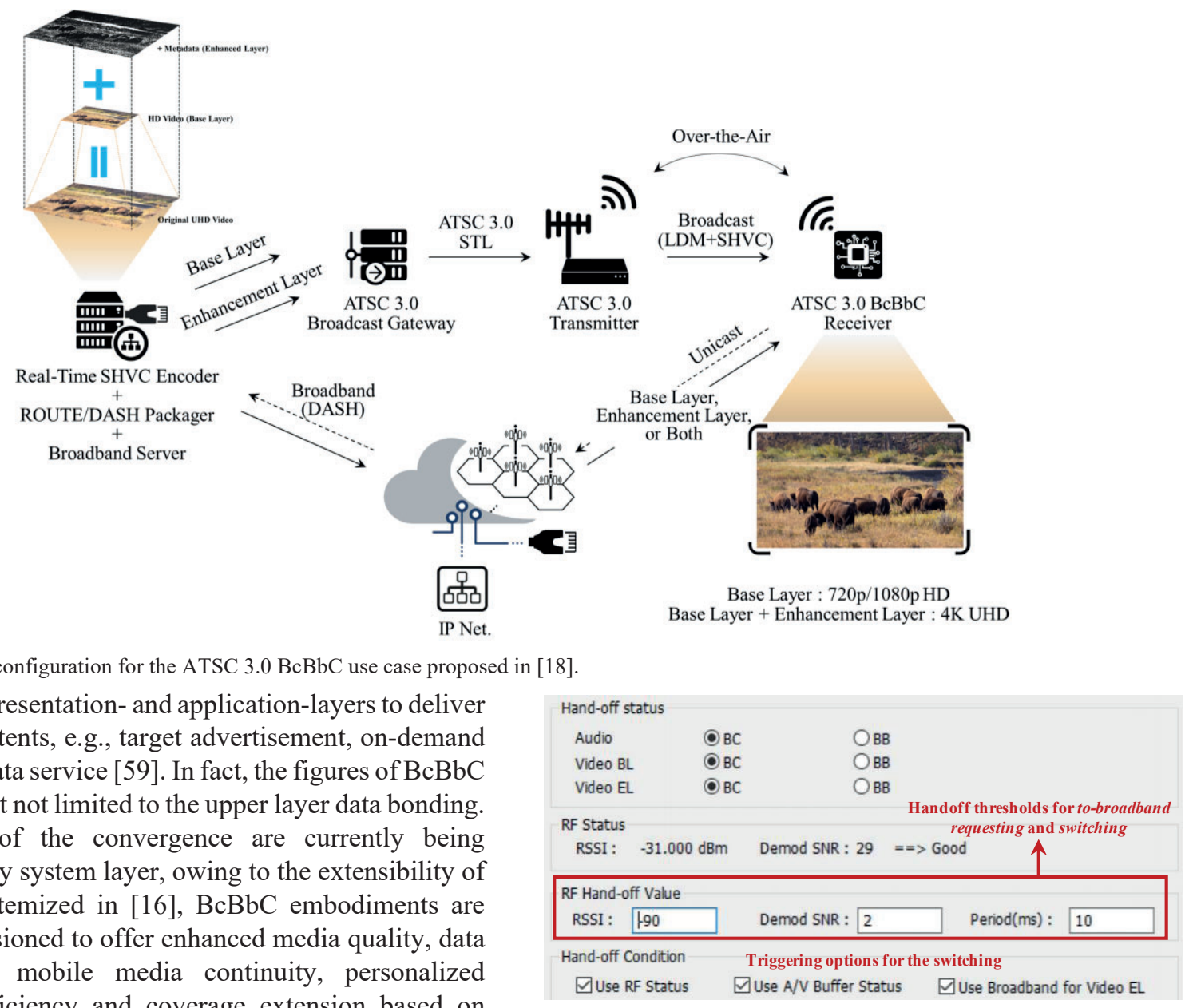

Fig. 13. Equipment configuration for the ATSC 3.0 BcBbC use case proposed in [18]

be embodied in presentation- and application-layers to deliver personalized contents, e.g., target advertisement, on-demand supplementary data service [59]. In fact, the figures of $\mathrm{BcBbC}$ would include but not limited to the upper layer data bonding. Diverse forms of the convergence are currently being discussed in every system layer, owing to the extensibility of ATSC 3.0. As itemized in [16], BcBbC embodiments are specifically envisioned to offer enhanced media quality, data traffic stability, mobile media continuity, personalized service, cost efficiency and coverage extension based on tower overlaying. Those features are expected to play versatile roles in a wide range of verticals, more than remaining in the $\mathrm{M} \& \mathrm{E}$ industry. In this end, the future ecosystem could be assisted by the $\mathrm{BcBbC}$ evolution in many aspects, such as intelligent traffic system (ITS), mobile positioning, and so on.

One rising example of $\mathrm{BcBbC}$ is seamless mobile streaming based on dual connectivity. As aforementioned in Section I and II, ATSC 3.0 is strongly anticipated to bring mobile DTT service of rich, over-HD quality videos into the picture. This mobile-supporting feature is seen further promising when we remind the drastic advance of automotive technology. However, there undesirable shadowing and coverage gaps, e.g., a tunnel, would still exist and hence may lay some temporal stream loss. If more than $4 \mathrm{~K}$ UHD quality is intended, such connection losses will be more significant because more than $10 \mathrm{~dB}$ SNR is typically required to receive over-15 Mbps ATSC 3.0 stream successfully. To resolve this problem, [18] and [19] proposed an idea to use a broadband link as a backup channel.

In the proposal [18], the convergence has been accomplished in transport- and application-layers. In parallel to the broadcast-side transmission, the studio was designed to upload timely segments of the same (live-encoded) video program streams to the on-line server also ${ }^{8}$. On the other hand, the video decoder application installed to an ATSC 3.0

8 At the IP-multiplexing component, encoded video stream was duplicated and then in parallel encapsulated into two different formats at the same time. UDP/IP over Dynamic Adaptive Streaming over HTTP (DASH) was used for the broadband path, and TCP/IP over MPEG Media Transport
Fig. 14. A controller software to monitor the RF signal status and determine the switching between broadcast and broadband connections [18].

receiver was implemented to request an apt video segment whenever the receiver is about to lose ATSC 3.0 signal.

To this end, the receiver keeps monitoring the statuses of the physical channel (i.e., SNR and received signal strength) and video buffer. Should be noted, the requesting physically lags to the reception of the broadcast signal and there also exists a negligible round-trip delay in the broadband delivery. The broadband requests were therefore executed in somewhat a conservative manner, prior to the expected broadcast signal loss. That is, the threshold SNR which triggers the request was set to be reasonably higher than the $\mathrm{ToV}$ of the broadcast signal.

The video segments received via broadband linkage were registered into the buffer, and then the receiver became able to keep the playback stable even while the ATSC 3.0 signal is blocked out. Conversely, the receiver was led to switch back into the broadcast link seamlessly (i.e., to idle the requesting) whenever ATSC 3.0 physical-layer connectivity is recovered. Note here that the handoffs to broadband were executed opportunistically and temporally, which therefore eased the users' financial burden for using paid networks. Keeping in mind the difference of end-to-end latency between broadcast and broadband deliveries, it was requested the packets whose presentation times precede to that of the 


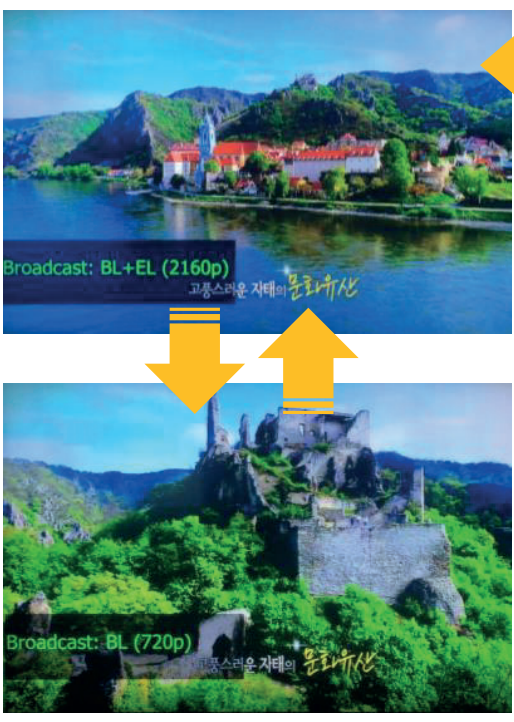

(a)

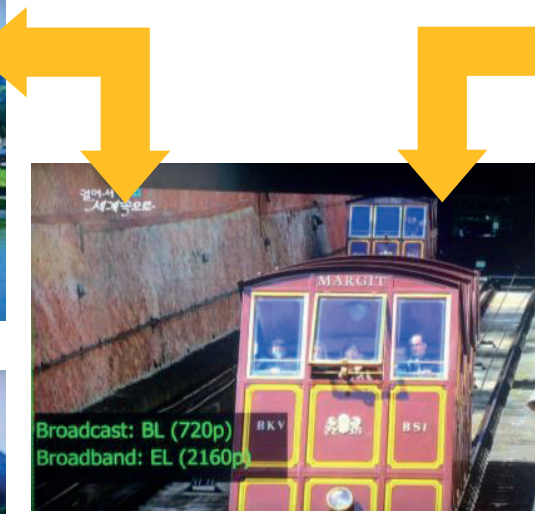

(b)

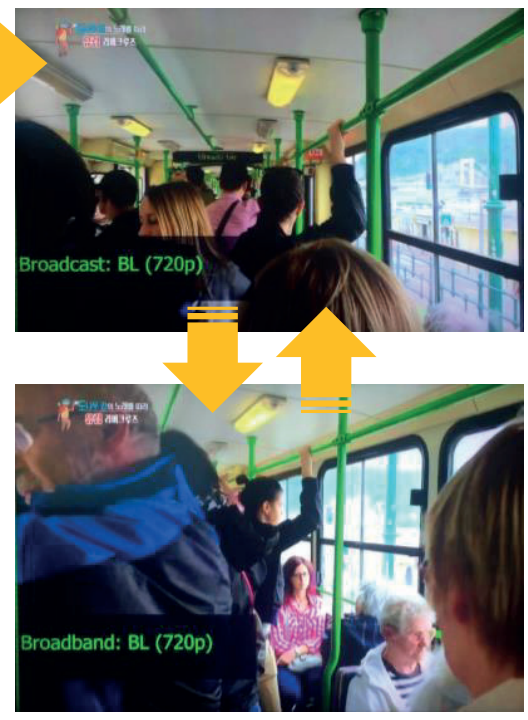

(c)

Fig. 15. Field trial instants in [18]: (a) Seamless switching between HD and UHD in broadcast-only mode, (b) hybrid service combining base layer from broadcast and enhancement layer from broadband, and (c) seamless switching between broadcast and broadband networks for HD only.

via-broadcast packet received at the triggered instant. To this end, the presentation time offset was heuristically determined by experiments.

Notably, the idea for BcBbC-assisted seamless mobile has been further extended decently, by being combined with scalable video coding (SVC). Precisely speaking, the implementation in [18] aimed at providing a scalable service based on H.265 Scalable High-Efficiency Video Codec (SHVC) ${ }^{9}$. A 720p HD service was encoded to be the SHVC base layer, and the SHVC enhancement layer consisted of metadata that supplement the base layer stream to elevate the video quality into $4 \mathrm{~K} \mathrm{UHD}^{10}$.

This figure of plugging SHVC into the proposed $\mathrm{BcBbC}$ system allowed even a $4 \mathrm{~K}$ UHD content to be seamlessly streamed in mobile environments. Where the base and enhancement layers were loaded into different PLPs, the receiver was able to combine the packets from both broadcast and broadband channels depending on the broadcast signal availability. For example, as shown in Fig. 15(b), base layerover-broadcast and enhancement layer-over-broadband packets could be combined to rebuild 4K UHD when the broadcast channel quality was good enough to decode the robust PLP containing base layer but not sufficient to decode the enhancement layer's PLP. Worth to mention, the user can choose whether to pursue maintaining the enhanced-quality video or to remain at base-quality service, depending on its affordability.

To expand the coverage more, this system was built on top of a cross-layer combination of SHVC+LDM [61]. The base and enhancement layers were there conveyed by CL and EL PLPs, respectively. The layered, hierarchical architectures of those two gears in essence fit into each other, and their engagement thereby lubricates a switching between base- and enhanced-quality services. In addition, a bit-reduction gain from SHVC and a spectral efficiency gain from LDM synergistically improve the system coverage of broadcast itself. This enhancement fortunately reduces the possibility to handover both base and enhancement layers entirely to broadband, hence bringing better economic efficiency to users.

A real-field practice of this convergence was demonstrated in Jeju Island, South Korea, by using a public LTE-A Pro network for the broadband part. Through the field-test carried out in 2019, it was well-verified that $\mathrm{BcBbC}$ guarantees a stable experience to on-vehicle users. In addition, the reliability gain of this $\mathrm{BcBbC}$ concept has been as well theoretically proved in [19].

\section{B. Traffic Stability and NOMA in Convergence Perspective}

Although 5G enhanced mobile broadband (eMBB) has raised the net throughput capability impressively, rapidly increasing demands on massive video data are still a nuisance to cellular networks. DTT networks have again been explored in this context, to offload the traffic upon its super-efficient point-to-multi-point (PTM) capability. Several groups of manufacturers are already in progress to develop the broadcast-offloading solutions, particularly concentrating on medium access control (MAC) layer functionalities.

Beyond the idea of traffic offloading, the convergence further down to the physical-layer level has been introduced as well [16], [17], [42], [62], [63]. The idea of physical-layer level cooperation emerged from an expectation that it would create new market opportunities. Specifically, broadcasters are envisioning an infra-leasing business based on tower overlay. At the broadband carrier's perspective, routing highpower high-towers (HPHTs) of the DTT network is an efficient option to reduce operating and capital expenditures required for widening its service coverage. If this physicallayer convergence is achieved above the advance of cloudbased network orchestration technologies, the $\mathrm{BcBbC}$ could
${ }^{9}$ Note that this development was fully compliant to ATSC 3.0 standard. The video specifications of ATSC 3.0 have adopted SHVC as well as HEVC.

${ }^{10}$ Following the terminologies in SHVC, a base layer is defined to be the smallest subset of a video content representing the lowest quality product. An enhancement layer (multiple enhancement layers can exist in principle, but up to one enhancement layer is allowed in ATSC 3.0) on the other hand indicates the larger, metadata subset which references the base layer. SHVC is designed in a hierarchical manner so that a better-quality video can be retrieved by combining base and enhancement layers during decoding. 


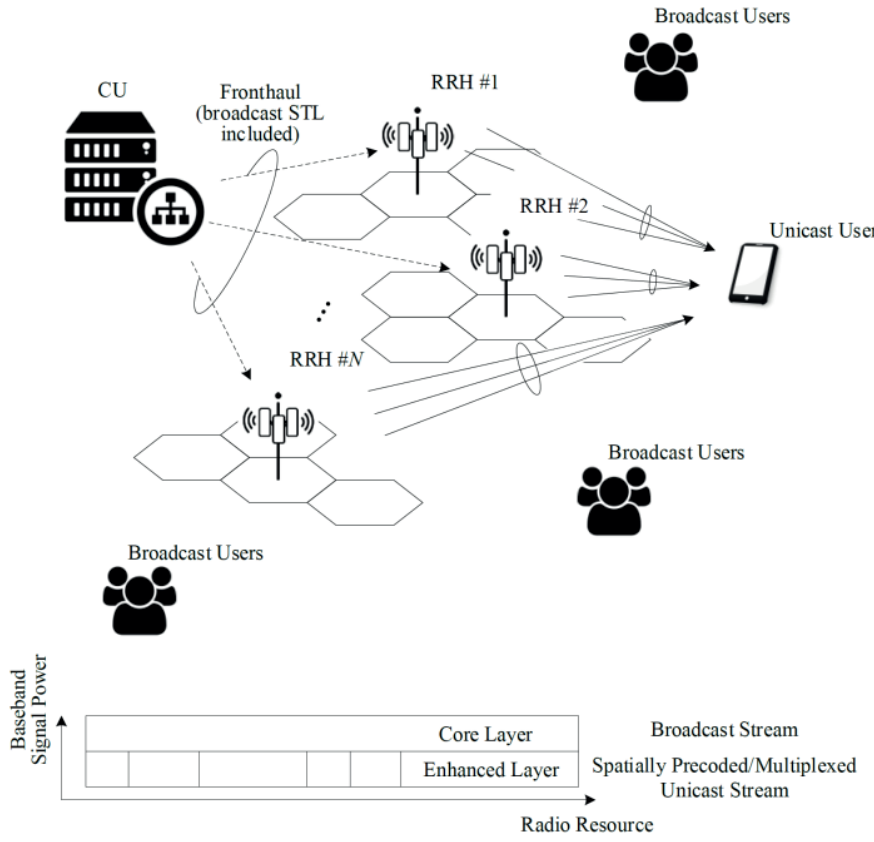

Fig. 16. Conceptual description in [42] for the C-RAN with BMUST.

come with an organically integrated network that can coordinate HPHTs and low-power low-towers (LPLTs) at every moment.

When we turn back to the fundamentals, $\mathrm{BcBbC}$ can be viewed as cooperation between broadcast and unicast transmissions. From this end, some pioneer works have analyzed the theoretical gain which can be obtained by a conjunction between broadcasting and unicasting. Those studies have illustrated abstract figures which can pave the way toward the convergence below Layer-3.

In particular, [42], [62]-[66] have focused on the usage of NOM for co-transmitting broadcast and unicast messages jointly. Let us henceforth refer to this class of NOM/NOMA as broadcast/multicast and unicast superposed transmission (BMUST), as in [63]. Those works have pointed out the possible gain from BMUST amplifying the benefit of $\mathrm{BcBbC}$, by underlining the throughput advantage from NOM. In addition, BMUST has a desirable edge at scheduling simplicity, because BMUST does not require a user pairing unlike MUST solely for unicasting.

The approach of [62] would especially be interesting to service and network providers. To take the offloading gain into account, [62] has brought the popularity of each content into the picture. Accordingly, the selection of the content set that is transmitted over broadcast channel, namely a broadcast content profile $(B C P)$, has been optimized to maximize the stochastic throughput (i.e., successful delivery rate) of the network. Alongside this, the BMUST frame structure has been proposed to convey broadcast and unicast signals by CL and EL pipes, respectively. Two different MAC policies have then been considered: (1) A generous $M A C$ that allows transmitting the broadcast contents via unicast; and (2) an offloading-oriented MAC that restricts the system not to use unicast transmissions for delivering the broadcast contents.

In turn, closed-form formulas for the optimal BCP selection strategy have been analytically derived in a
TABLE I. BICM COMPONENTS OF ATSC 3.0, FEMBMS, AND 5G NR

\begin{tabular}{|c|c|c|c|}
\hline & $\begin{array}{c}\text { FEC for } \\
\text { data channel }\end{array}$ & $\begin{array}{c}\text { FEC for } \\
\text { control channel }\end{array}$ & Constellation \\
\hline ATSC 3.0 & LDPC codes & $\begin{array}{c}\text { S/P LDPC } \\
\text { codes }\end{array}$ & NUC \\
\hline FeMBMS & Turbo codes & $\begin{array}{c}\text { Convolutional } \\
\text { codes }\end{array}$ & QAM \\
\hline 5G NR & LDPC codes & Polar codes & QAM \\
\hline
\end{tabular}

probabilistic form. The optimal strategy has been found to be dependent on network density, network access load, bit-rate and popularity of each content, and so on. This optimal BCP selection has been shown to be definitely beneficial than the maximum popularity selection and uniform probability selection strategies regardless of the popularity distribution. Numerical results in [62] have verified a significant offloading gain of $\mathrm{BcBbC}$, and BMUST has also been shown to achieve over $170 \%$ of network throughput gain over orthogonal multiplexing-based $\mathrm{BcBbC}$.

On the other hand, [42] has delved into the radio transmission chain more. Unlike the stochastic geometry framework of [62], [42] has considered a deterministic system topology. Instead, the coordinated multi-point (CoMP) signal processing has been addressed to facilitate BMUST within cloud-radio access networks (C-RANs). As also been pointed out in [16], the centralized natures of broadcasting and C-RAN have been highlighted to be fit well with each other. The effect of broadcast signal coexistence has been carefully investigated for both central unit-to-frontend (i.e., fronthaul) and frontend-to-user connections, and reflected to the compressions over fronthauls and the spatial multiplexing over physical channels. Precisely, in [42], precoding and fronthaul compression for each remote frontend have been jointly optimized to maximize the sum-rate of unicast transmissions.

Note that every BMUST studies above have considered the CLI penalty that turns out when the CL decoding is failed. Impressively, BMUST has been shown still beneficial than the traditional radio access technologies despite the destructive CLI effect. Owing to this delightful throughput advantage, BMUST will turn out to be a super-appealing feature in the future $\mathrm{BcBbC}$ era, in which intimate convergences are realized in various system layers.

In addition to applications at the frontend transmissions, using LDM for wireless fronthauls/backhauls could also help to cope with resource scarcity. Spectrally efficient LDM transportations would possibly reduce the static resource occupation of wireless backhauls, and therefore would allow the users to experience better QoS, in average [67].

\section{BICM Comparison with FeMBMS and $5 G N R$}

Through the BICM chain, input data is protected by applying FEC and then mapped to constellation points. BICM chain, which is closely related to spectral-efficient and robust transmission, should be designed to meet the requirements of the systems.

Quasi error-free performance, e.g., block error rate (BLER) $\leq 10^{-6}$, is required for broadcasting systems, and therefore 
TABLE II. PERFormanCE OF ATSC 3.0, FEMBMS, AND 5G NR $[75]$

\begin{tabular}{|c|c|c|c|c|}
\hline $\begin{array}{c}\text { Code } \\
\text { Rate }\end{array}$ & $\begin{array}{c}\text { ATSC } \\
(n=64800)\end{array}$ & $\begin{array}{c}\text { ATSC } \\
(n=16200)\end{array}$ & $\begin{array}{c}5 \mathrm{G} \text { NR } \\
(n=16200)\end{array}$ & $\begin{array}{c}\text { FeMBMS } \\
(k=6144)\end{array}$ \\
\hline $3 / 15$ & -4.31 & -3.80 & -3.98 & -3.48 \\
\hline $4 / 15$ & -2.87 & -2.37 & -2.52 & -2.16 \\
\hline $5 / 15$ & -1.66 & -1.33 & -1.38 & -1.36 \\
\hline $6 / 15$ & -0.48 & -0.30 & -0.35 & -0.28 \\
\hline $7 / 15$ & 0.32 & 0.56 & 0.52 & 0.63 \\
\hline $8 / 15$ & 1.20 & 1.36 & 1.50 & 1.48 \\
\hline $9 / 15$ & 2.00 & 2.19 & 2.23 & 2.35 \\
\hline $10 / 15$ & 2.81 & 2.96 & 3.05 & 3.23 \\
\hline $11 / 15$ & 3.63 & 3.81 & 3.87 & 4.05 \\
\hline $12 / 15$ & 4.51 & 4.70 & 4.73 & 4.93 \\
\hline $13 / 15$ & 5.54 & 5.75 & 5.77 & 5.98 \\
\hline
\end{tabular}

LDPC codes of length 16,200 and 64,800 bits are employed in both DVB-T2/S2 and ATSC 3.0 systems. During their standardization, it is focused on optimizing the performance of LDPC codes for the combinations of fixed code lengths and rates [68]-[75]. Contrary to DTT broadcasting systems, 3GPP, which is developing a standard for wireless communications, should support the high flexibility required for adapting to the channel quality and the size of the payload [76], [77]. For this reason, the flexibility in terms of code rate and length is the critical requirement in the design of the $\mathrm{BICM}$ chain for $3 \mathrm{GPP}$.

In 3GPP, further evolved multimedia broadcast multicast service (FeMBMS) is referred to as the most advanced technique for terrestrial broadcasting, which is based on eMBMS first standardized in Rel-9. FeMBMS is further evolved in Rel-16 in order to satisfy the 5G requirements [76] which requests large inter-site distance (ISD) up to $100 \mathrm{~km}$ and high mobility up to $250 \mathrm{~km} / \mathrm{h}$ for SFN. As a result, a new numerology, which uses $0.37 \mathrm{kHz}$ subcarrier spacing (SCS) and 300 us cyclic prefix (CP), is endorsed so that LTE-based $5 \mathrm{G}$ broadcast (i.e., evolved FeMBMS) can meet the requirements for $5 \mathrm{G}$ multimedia broadcast/multicast service (MBMS). However, LTE-based 5G broadcast is based on FeMBMS which employs turbo codes and convolutional codes as its FEC schemes, and therefore it is considered as limitations of FeMBMS from the viewpoint of physical-layer performance.

One innovative technology adopted in 5G new radio (NR) physical-layer is to employ LDPC codes and polar codes as FEC schemes for protecting data and control channels. As a result, 5G link-level performances are better than those of LTE which uses turbo codes and convolutional codes as its physical-layer FEC schemes. Future terrestrial broadcasting in $3 \mathrm{GPP}$ based on NR physical-layer can take advantage of not only recent enhancements for terrestrial broadcasting in Rel-16 but also the advanced NR BICM chain. Table I represents components of the BICM chain of ATSC 3.0, FeMBMS, and 5G NR.

Link-level performances of FEC schemes employed for data channels of ATSC 3.0, FeMBMS, and 5G NR are compared in Table II, which represents the required SNRs to achieve BLER $=10^{-2}$ over the additive white Gaussian noise

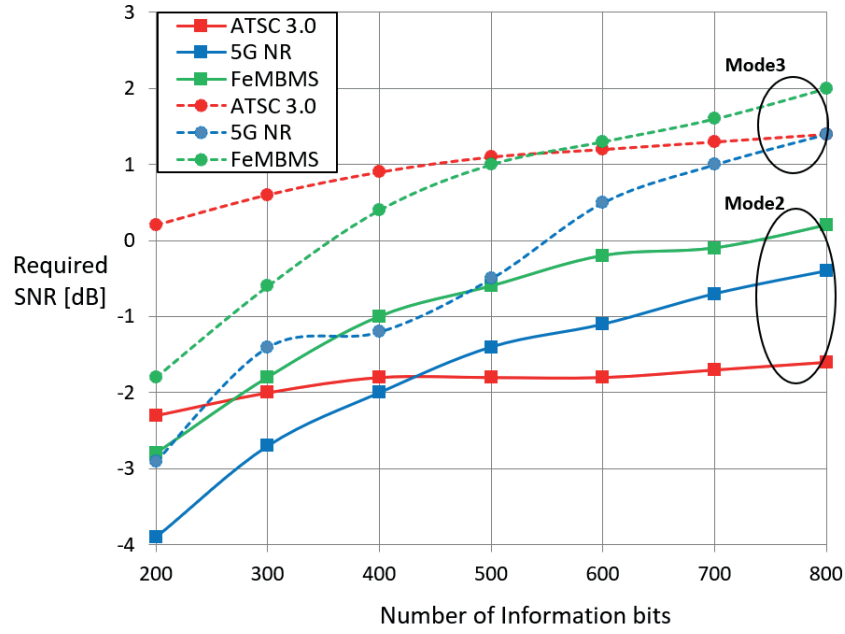

Fig. 17. Performance of BICM chains for control information: Comparisons with L1-protection mode 2 and mode 3 of ATSC 3.0.

(AWGN) channels. Since the number of information bits $k$ supported by the 5G NR LDPC codes is limited to 8448, LDPC codes are generated by lifting base graph1 (BG1) with a larger size than that defined in the spec. [77]. Table II shows that ATSC 3.0 with 64,800 bits-length LDPC codes has the best performance thanks to dedicatedly designed long LDPC codes. In addition, the performance advantage of ATSC 3.0 LDPC codes gets better compared to FeMBMS when target BLER is less than $10^{-6}$ because turbo codes suffer from error floor phenomenon. Moreover, when transmitting data with high-order modulations (HOMs), ATSC 3.0 has a strictly better performance than FeMBMS and 5G NR employing QAM constellation due to the shaping gain of the NUC [7]. The performance gain from NUC reaches up to about $1 \mathrm{~dB}$ when the modulation order is 256 .

In order to properly decode the data at the receiver, the control information should be decoded properly because it includes essential information for receiving data channels. In the case of FeMBMS, it is transmitted in a cell acquisition subframe (CAS) [78]. In the case of ATSC 3.0, it is called layer-1 (L1) signaling which is divided into L1-basic and L1detail. These signaling information are strongly protected by FEC schemes, unlike the ones used for data channels. FeMBMS and 5G NR have adopted convolutional codes and polar codes for protecting control information, respectively. On the other hand, ATSC 3.0 makes use of the same LDPC codes adopted for data channels with information shortening and parity puncturing, which is necessary to support the required number of bits and code rate for control channels [7]

Link-level performance of FEC schemes for control channels of ATSC 3.0, FeMBMS, and 5G NR are compared in Fig. 17, which represents the required SNRs to achieve BLER $=10^{-2}$ over AWGN channels. To ease the comparison, the number of information bits is chosen from 200 to 800 and the number of codeword bits is determined to support mode 2 and mode 3 of L1-detail protection [73], [7]. Note that the code rates and net codeword lengths of FeMBMS and $5 \mathrm{G}$ NR frames were controlled to be the same with ATSC 3.0 frames for each comparison.

As shown in Fig. 17, the performance of ATSC 3.0 is rather uniform according to the number of information bits. When 
the number of information bits is small, the performance of ATSC 3.0 is the worst because massive shortening and puncturing are inevitable to support the small number of signaling bits. Even though the patterns for shortening and puncturing is delicately designed for the given length-16,200 LDPC codes, it cannot reach up to the performance of polar codes and convolutional codes. As the number of information bits increase, however, the BLER performance of $5 \mathrm{G}$ NR and FeMBMS is much more degraded than that of ATSC 3.0 because 5G NR polar codes is designed up to length-1024 and convolutional codes cannot have benefit of long code-length. It means that the physical-layer performance of the FEC scheme for L1 signaling is comparable with that of polar codes and convolutional codes in 5G NR and FeMBMS and it can be improved by designing new dedicated LDPC codes for protecting L1 signaling.

\section{FIELD VERIFICATION}

A number of active research groups have examined the end-to-end system performance of ATSC 3.0 to provide a practical guideline for implementation and service [8]. Those verifications were not limited to computer simulation and laboratory test, but also encompassed the field trials in real environments. In particular, [9] and [3] have drawn exhaustive evaluations for the reliability of every ModCod combination in ATSC 3.0 physical-layer ${ }^{11}$. Whereas the baseline performance based on non-LDM transmissions have been addressed in [9] and [3], the throughput/reliability gain of LDM over TDM has additionally been measured and reported in [79]. Those results have been officially published in [8] to be the reference measures. This section gives a brief review of those celebrated results, particularly on field test results. The revisited results may be especially informative to potential network organizers, as those offer an intuition upon the practice of ATSC 3.0 physical-layer.

\section{A. Reliability of ATSC 3.0 Physical-Layer}

The reports [9] and [8] provide an exhaustive list of the ToVs for ATSC 3.0 transmissions measured in several representative stationary channel models. Motivated by [80], the laboratory experiments were carried out over three reference channel models: An AWGN channel; a Rician channel with a single line-of-sight component and 19 multipaths (RC20); and a Rayleigh channel with 20 multipath components (RL20). To follow-up with coherency, the experiments continued in the field measured the results in carefully chosen reception points whose channel profiles were seen $A W G N$-like, RC20-like, or RL20-like.

The field experiments of [9] took place in Jeju Island, South Korea. To this end, the full-chain studio and transmission facilities were constructed for ATSC 3.0 broadcasting (See Fig. 18). For the receiver-side, a professional testing vehicle was built as shown in Fig. 19. A specialized measurement system was implemented therein, so as to integrate professional ATSC 3.0 physical-layer receiver, spectrum analyzer, noise generator, low noise amplifier, antenna elevator and rotor, media decoder, Global Positioning System (GPS) receiver, and signal attenuator

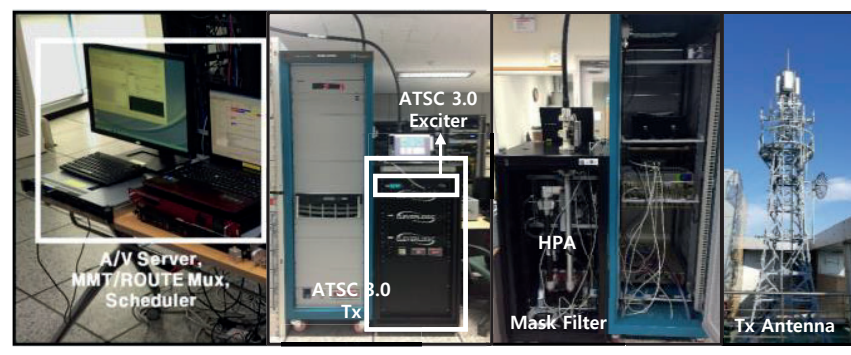

Fig. 18. Facility configurations: Studio/transmitter-side infrastructure [9].

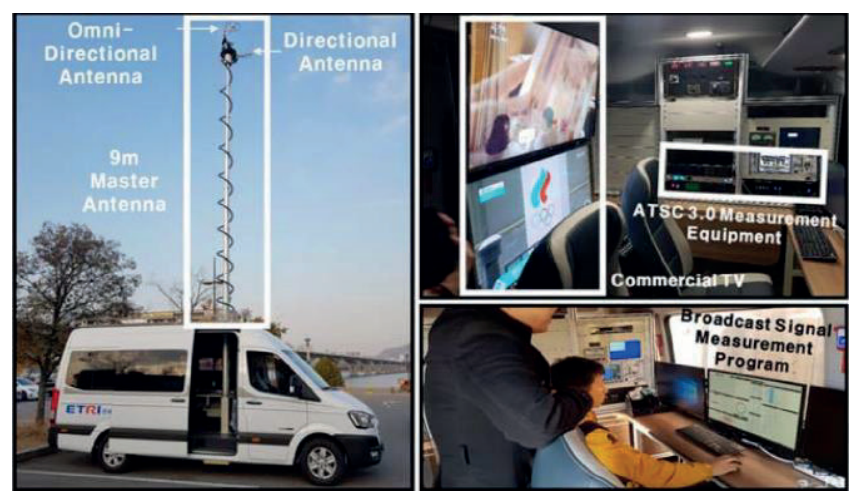

Fig. 19. Facility configurations: Customized vehicle for measurements [9].

under a unified software controller so-called the Integrated Measurement Analysis System (IMAS). Using this measurement system, decoding errors were recoded bit-bybit and frame-by-frame at every instant, together with the information of the overall reception environment profile.

The signal robustness of each ModCod combination was quantified by its ToV, the SINR at least required to assure QEF. Complying with ATSC's recommendations for fixed service, [9] measured the ToVs at the SNR points where the frame error rate (FER) equals to $10^{-4}$ in approximate. Through the presented results, [9] demonstrated that a variety of transmission capacities in ATSC 3.0 are feasible in the real world. Every BICM configuration of ATSC 3.0 stably delivered the guaranteed throughput if sufficient signal strength was acquired. For example, an HD video with about 1.1 Mbps could be successfully received even at $-3 \mathrm{~dB}$ SNR environment. While the coverage was correspondingly reduced, over $50 \mathrm{Mbps}$ transmissions were successfully verified in the field also. As for the promising use case, $4 \mathrm{~K}$ UHD, around $15 \mathrm{Mbps}$ delivery was available at $12.8 \mathrm{~dB}$ $14.5 \mathrm{~dB}$ SNR, depending on the multipath profile.

By and large, the ToV was shown in [9] to be increased by LDPC code rate and constellation order. However, an interesting property was found at the same time: Combining the high-rate LDPC codes with the low-order constellation, e.g., QPSK, sometimes could not protect the signal so well as the other ModCod options which achieve the analogous throughput. This aspect was once discussed during the standardization, and in this regard, the physical-layer specification [7] of ATSC 3.0 has not included the combinations of high-rate LDPC codes and low-order constellations in the mandatory ModCod set.

Such performance degradation turned out significant especially in fading channel environments, as shown in Fig. 20 [9]. Can be noticed from the result in Fig. 20, the 


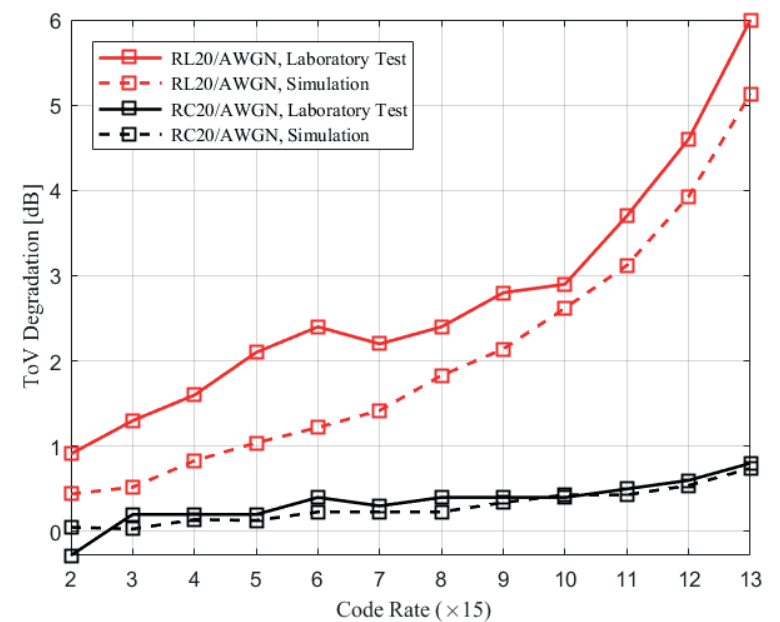

Fig. 20. Fading channel impact on ToV with respect to LDPC code rates (QPSK-modulated) [9]

degradation was found more significant in RL20 channel case than that of RC20 case. Interpreting this result into a relative vulnerability against harsh channel environments, it may be incurred that the combinations of high-rate LDPC+low-order constellation would not be adequate for mobile channels although they could show a good performance in AWGN or mild multipath channels.

\section{B. Field Comparison between LDM and TDM in ATSC 3.0}

1) Fixed Reception Results

Based on the decent facility construction in Jeju Island, the field feasibility of ATSC 3.0 LDM was also validated. The report [79], [81] demonstrated that LDM truly achieves substantial coverage and capacity gains over TDM in practice as was touted widely. A reasonable UEP scenario was considered to serve two video contents with different bit-rates A 720p HD video and a 4K UHD video, both HEVC-coded, were broadcasted over-the-air via the $\mathrm{CH} 50$ (centered at 689 $\mathrm{MHz}$ ) radio channel. Both video services were contained in every physical-layer frame, where each video service was loaded into its dedicated PLP. The detailed physical-layer configurations are described in Table III.

Table IV recalls the field comparison results presented in $[81]^{12}$. To be noted, ATSC 3.0 LDM assured better reliability than TDM for both HD and UHD services simultaneously, where the throughput of each PLP was set analogous between the LDM and TDM cases. LDM precisely attained about 3.3 $\mathrm{dB}$ ToV gain over TDM for the HD service and reduced nearly $2.2 \mathrm{~dB}$ of ToV for the UHD service.

Such superiority on error protection capability was reflected to a coverage extension in quality. As for the rooftop receptions conducted across the 40 selected test points in Jeju City, LDM CL reception was succeeded at every test point while $5 \%$ of the test points failed at retrieving the HD service in TDM frames. In addition, the UHD video in LDM EL was available at $72.5 \%$ test points while the TDM counterpart showed a $70 \%$ success rate.

Indoor environments were shown more challenging, whereby the indoor RSS (averaged over 20 test sites) was
TABLE III. ATSC 3.0 PHYSICAL-LAYER PARAMETERS USED IN LDM/TDM FIELD COMPARISON TESTS OF [81] AND [82]

\begin{tabular}{|c|c|c|c|}
\hline \multirow{2}{*}{\multicolumn{2}{|c|}{ Frame Length }} & LDM & TDM \\
\hline & & \multicolumn{2}{|c|}{$251.33 \mathrm{~ms}$} \\
\hline \multicolumn{2}{|c|}{ Occupied Bandwidth } & \multicolumn{2}{|c|}{$5.832844 \mathrm{MHz}$} \\
\hline \multicolumn{2}{|c|}{ Time Interleaver } & \multicolumn{2}{|c|}{ CTI, 1024-sample depth } \\
\hline \multicolumn{2}{|c|}{ Frequency Interleaver } & \multicolumn{2}{|c|}{$\mathrm{ON}$} \\
\hline \multirow{9}{*}{$\begin{array}{l}\text { HD } \\
\text { Service }\end{array}$} & & CL & $1^{\text {st }}$ Subframe \\
\hline & FFT Size & $16 \mathrm{k}$ & $8 \mathrm{k}$ \\
\hline & Guard Interval & \multicolumn{2}{|c|}{148.15 us (GI5 1024) } \\
\hline & Pilot Pattern & \multicolumn{2}{|c|}{ SP Dx $=6, \mathrm{Dy}=2$} \\
\hline & $\begin{array}{l}\text { \# of Payload } \\
\text { Symbols }\end{array}$ & 98 & 75 ( $38 \%$ of each frame) \\
\hline & Outer Code & \multicolumn{2}{|c|}{$\frac{1}{\mathrm{BCH}}$} \\
\hline & Inner Code & $\begin{array}{l}\text { 4/15 LDPC (64K } \\
\text { Length) }\end{array}$ & $\begin{array}{c}\text { 11/15 LDPC (64K } \\
\text { Length) }\end{array}$ \\
\hline & Constellation & \multicolumn{2}{|c|}{ QPSK } \\
\hline & Data Rate & $2.59 \mathrm{Mbps}$ & $2.75 \mathrm{Mbps}$ \\
\hline \multirow{8}{*}{$\begin{array}{l}\text { UHD } \\
\text { Service }\end{array}$} & & EL & $2^{\text {nd }}$ Subframe \\
\hline & FFT Size & $16 \mathrm{k}$ & $32 \mathrm{k}$ \\
\hline & Guard Interval & \multicolumn{2}{|c|}{148.15 us (GI5 1024) } \\
\hline & $\begin{array}{c}\text { \# of Payload } \\
\text { Symbols }\end{array}$ & 98 & $30(62 \%$ of each frame $)$ \\
\hline & Inner Code & $\begin{array}{c}\text { 10/15 LDPC (64K } \\
\text { Length) }\end{array}$ & $\begin{array}{c}\text { 12/15 LDPC (64K } \\
\text { Length) }\end{array}$ \\
\hline & Outer Code & \multicolumn{2}{|c|}{$\mathrm{BCH}$} \\
\hline & Constellation & $64 \mathrm{NUC}$ & $256 \mathrm{NUC}$ \\
\hline & Data Rate & $19.56 \mathrm{Mbps}$ & $19.90 \mathrm{Mbps}$ \\
\hline \multicolumn{2}{|c|}{ Injection Level } & $-4 \mathrm{~dB}$ & - \\
\hline
\end{tabular}

TABLE IV. LDM/TDM FIELD COMPARISON RESULTS PRESENTED IN [81] (FIXED RECEPTION, AVG. SigNAL STRENGTH $=-63.53 \mathrm{dBm}$ )

\begin{tabular}{|c|c|c|c|}
\hline \multicolumn{2}{|c|}{} & $\begin{array}{c}\text { Throughput } \\
{[\mathrm{Mbps}]}\end{array}$ & Measured ToV [dB] \\
\hline \hline \multirow{2}{|c|}{$\begin{array}{c}\text { HD Service } \\
(720 \mathrm{p})\end{array}$} & LDM CL & 2.59 & 2.29 \\
\cline { 2 - 4 } & ${\text { TDM } 1^{\text {st }} \text { Subframe }}^{2}$ & 2.75 & 5.77 \\
\hline $\begin{array}{c}\text { UHD Service } \\
(4 \mathrm{~K})\end{array}$ & LDM EL & 19.56 & 20.61 \\
\cline { 2 - 4 } & TDM 2 $2^{\text {nd }}$ Subframe & 19.90 & 22.91 \\
\hline
\end{tabular}

nearly $19 \mathrm{~dB}$ lower than the average RSS measured at rooftop reception sites. The average RSS of the indoor case was $82.33 \mathrm{dBm}$ and that of the rooftop case was $-63.53 \mathrm{dBm}$. As for the indoor experiments, LDM brought $15 \%$ p more places into the HD coverage, compared to the TDM counterpart. Due to a significant signal strength loss from penetration, only $10 \%$ of the test sites were available of the UHD PLP, for both LDM and TDM cases.

2) Mobile Reception Results

As once mentioned above, one of the most featured use cases over ATSC 3.0 LDM is the coexistence of mobile and fixed services within the same physical-layer frame. The HD service configurations in Table III were designed to be a versatile carrier that could serve mobile recipients also, in addition to indoor and coverage-edge users or the global service recipients residing between the coverages of locally inserted services [30], [39].

Aside from the comparison for fixed receptions, the mobile reliability was compared in the same project [81] (and the related works [82], [79]). Results exhibited pragmatic evidence whereby touted an excellent mobile coverage extension opportunity of using LDM.

To summarize the experiment of [82], an ATSC 3.0 measurement system mounted in the test vehicle recorded every instant decoding errors that arose during each drive across the downtown of Jeju City. The configurations in

${ }^{12}$ At each measurement point, the ToV was measured by injecting artificial AWGN until the reception cannot maintain an errorless video playback over 60 seconds. 


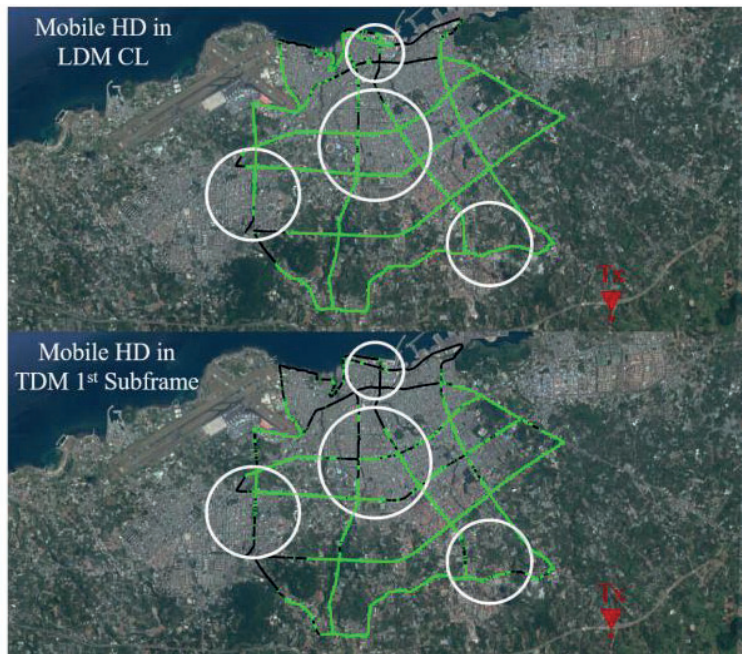

Fig. 21. Reception performance comparison between the mobile services over LDM and TDM: Reception success (green), reception failure (black). LDM case (upper), TDM case (lower) [82].

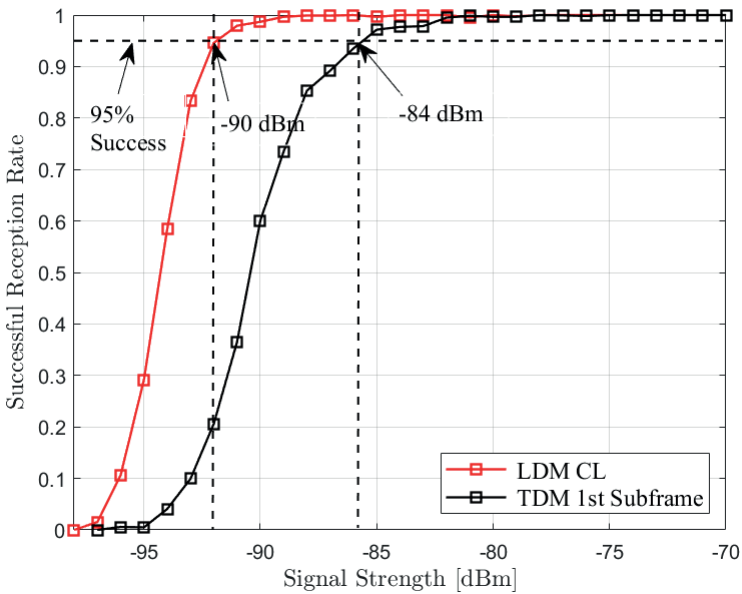

Fig. 22. Field comparison test result: ESR curves for ATSC 3.0 mobile LDM and TDM services [82].

Table III were reused for those experiments. At every second, physical-layer frame errors were recorded in pairs with the location (GPS) and RSS information at that instant ${ }^{13}$. In turn, every reception success or failure could be marked on a map as presented in Fig. 21.

As shown in Fig. 21 clearly, applying LDM evidently reduced the reception failures, compared to TDM. Such an improvement was especially notable in the areas pointed by white circles. It was shown that most of the points of interest were brought into the coverage by applying LDM, despite the challenging penalty from mobility. This advantage was also well evaluated in terms of the successful reception rate. To this end, the erroneous second ratio (ESR)-5 criterion was used, which is recommended by the International Telecommunication Union-Radiocommunication Sector (ITU-R) guideline. Accordingly, Fig. 22 plots the ESR curve that rearranged the results of [82]. According to Fig. 22, the usage of LDM could reduce the RSS requirement by $6 \mathrm{~dB}$, subject to $95 \%$ success of HD service playback during the driving time.

As well, there were many other efforts to verify the mobile feasibility of ATSC 3.0 and the gain of LDM. For instance,
[3] thoroughly presented the mobile performance of every ATSC 3.0 ModCod recommended in [8], which was measured by computer simulations (laboratory tests were also included partly) under a 6-tap typical urban (TU-6) channel. In the same paper, comprehensive discussions on the effect of various system components, e.g., pilot boosting, LDM transmission, time-domain windowing, and SFN operation, were carried out for ATSC 3.0 mobile service. [83] figured out the effect of transmit diversity code filter set (TDCFS) filtering into an SFN-based mobile service over LDM, through field tests in Jeju Island. As for a theoretical approach, [84] proved that LDM could cope better with receiver mobility than TDM when multiple services are intended in a single RF channel.

\section{CONCLUSION}

This paper introduced several selected features of ATSC 3.0 that could play a prominent role in the near future. This investigation encompassed the optional technologies defined in ATSC 3.0 physical-layer which can improve single frequency network integrity, enable additional features in other domains, or further widen the throughput capability. Majorly focused on the extensibility of ATSC 3.0, we also elaborated on possible inter-network cooperation with broadband and cellular systems.

\section{REFERENCES}

[1] L. Fay et al., "An overview of the ATSC 3.0 physical layer specification," IEEE Trans. Broadcast., vol. 62, no. 1, pp. 159-171, Mar. 2016

[2] R. Chernock et al., "ATSC 3.0 next generation digital TV standard An overview and preview of the issue," IEEE Trans. Broadcast., vol. 62, no. 1, pp. 154-158, Mar. 2016.

[3] S. Ahn et al., "Mobile performance evaluation for ATSC 3.0 physical layer modulation and code combinations under TU-6 channel," accepted for publication in IEEE Trans. Broadcast

[4] D. Gómez-Barquero and M. W. Caldwell, "Television spectrum incentive auctions in the U.S.: Trends, challenges, and opportunities," IEEE Commun. Mag., vol. 53, no. 7, pp. 50-56, Jul. 2015.

[5] L. Michael and D. Gómez-Barquero, "Bit-interleaved coded modulation (BICM) for ATSC 3.0," IEEE Trans. Broadcast., vol. 62 , no. 1, pp. 181-188, Mar. 2016.

[6] Advanced Television Systems Committee, ATSC Standard: A/321, System Discovery and Signaling, Doc. A/321, Mar. 2016

[7] Advanced Television Systems Committee, ATSC Standard: A/322, Physical Layer Protocol, Doc. A/322, Jan. 2020.

[8] Advanced Television Systems Committee, ATSC Standard: A/327, ATSC Recommended Practice: Guidelines for the Physical Layer Protocol, Doc. A/327, Jan. 2020.

[9] S.-I. Park et al., "Performance analysis of all modulation and code combinations in ATSC 3.0 physical layer protocol," IEEE Trans. Broadcast., vol. 62, no. 2, pp. 197-210, Jun. 2019.

[10] M. Earnshaw et al., "Physical layer framing for ATSC 3.0," IEEE Trans. Broadcast., vol. 62, no. 1, pp. 263-270, Mar. 2016.

[11] J. Lee et al., "Layered division multiplexing for ATSC 3.0: Implementation and memory use aspects," IEEE Trans. Broadcast., vol. 65, no. 3, pp. 496-503, Sep. 2019.

[12] D. He et al., "System discovery and signaling transmission using bootstrap in ATSC 3.0," IEEE Trans. Broadcast., vol. 62, no. 1, pp. 172-180, Mar. 2016.

[13] Advanced Television Systems Committee, ATSC Standard: A/331, Signaling, Delivery, Synchronization, and Error Protection, Doc. A/331, Jan. 2020. 
[14] W. Kwon et al., "The ATSC link-layer protocol (ALP): Design and efficiency evaluation," IEEE Trans. Broadcast., vol. 62, no. 1, pp. 316327, Mar. 2016.

[15] G. K. Walker et al., "ROUTE/DASH IP streaming-based system for delivery of broadcast, broadband, and hybrid services," IEEE Trans Broadcast., vol. 62, no. 1, pp. 328-337, Mar. 2016.

[16] D. Gómez-Barquero et al., "IEEE transactions on broadcasting special issue on: Convergence of broadcast and broadband in the $5 \mathrm{G}$ era,' IEEE Trans. Broadcast., vol. 66, no. 2, part 2, Jun. 2020.

[17] M. Simon et al., "ATSC 3.0 broadcast 5G unicast heterogeneous network converged services starting Release 16," IEEE Trans. Broadcast., vol. 66, no. 2, pp. 449-458, Jun. 2020

[18] J. Lee et al., "IP-based cooperative services using ATSC 3.0 broadcast and broadband," IEEE Trans. Broadcast., vol. 66, no. 2, part 2, Jun. 2020 .

[19] S. Ahn et al., "Cooperation between LDM-based terrestrial broadcast and broadband unicast: On scalable video streaming applications," accepted for publication in IEEE Trans. Broadcast.

[20] D. Gómez-Barquero et al., "MIMO for ATSC 3.0," IEEE Trans. Broadcast., vol. 62, no. 1, pp. 298-305, Mar. 2016.

[21] L. Stadelmeier et al., "ATSC 3.0 channel bonding," IEEE Trans. Broadcast., vol. 62, no. 1, pp. 289-297, Mar. 2016.

[22] T. Cover, "Broadcast channels," IEEE Trans. Inf. Theory, vol. 18, no. 1, pp. 2-14, Jan. 1972.

[23] R. Zhang and L. Hanzo, "A unified treatment of superposition coding aided communications: Theory and Practice," IEEE Trans. Wireless Commun., vol. 11, no. 7, pp.2628-2639, Jul. 2012.

[24] S. Vanka et al., "Superposition coding strategies: Design and experimental evaluation," IEEE Trans. Wireless Commun., vol. 11, no. 7, pp. 2628-2639, Jul. 2012

[25] Study on Downlink Multiuser Superposition Transmission (MUST) for LTE, document TR 36.859, 3GPP, Dec. 2015.

[26] L. Zhang et al., "Layered-division-multiplexing: Theory and practice," IEEE Trans. Broadcast., vol. 62, no. 1, pp. 216-232, Mar. 2016.

[27] S.-I. Park et al., "Low complexity layered division multiplexing for ATSC 3.0," IEEE Trans. Broadcast., vol. 62, no. 1, pp. 233-243, Mar. 2016.

[28] C. Regueiro et al., "LDM core services performance in ATSC 3.0," IEEE Trans. Broadcast., vol. 62, no. 1, pp. 244-252, Mar. 2016.

[29] E. Garro et al., "Layered division multiplexing with multi-radiofrequency channel technologies," IEEE Trans. Broadcast., vol. 62, no. 2, pp. 365-374, Jun. 2016.

[30] W. Li et al., "Using LDM to achieve seamless local service insertion and local program coverage in SFN environment," IEEE Trans. Broadcast., vol. 63, no. 1, pp. 250-259, Mar. 2017.

[31] J. Lee et al., "Multiple service configurations based on layered division multiplexing," IEEE Trans. Broadcast., vol. 63, no. 1, pp. 267-274 March 2017.

[32] L. Zhang et al., "Layered-division-multiplexing: An enabling technology for multicast/broadcast service delivery in 5G," IEEE Commun. Mag., vol. 56, no. 3, pp. 82-90, Mar. 2018.

[33] E. Garro et al., "Layered division multiplexing with distributed multiple-input single-output schemes," IEEE Trans. Broadcast., vol 65 , no. 1, pp. 30-39, Mar. 2019.

[34] L. Zhang et al., "Using layered-division-multiplexing to deliver multilayer mobile services in ATSC 3.0," IEEE Trans. Broadcast., vol. 65 no. 1, pp. 40-52, Mar. 2019

[35] L. Zhang et al., "Layered-division-multiplexing for high spectrum efficiency and service flexibility in next generation ATSC 3.0 broadcast system," IEEE Wireless Commun., vol. 26, no. 2, pp. 116 123, Apr. 2019.

[36] E. Garro et al., "Layered division multiplexing with co-located multiple-input multiple-output schemes," IEEE Trans. Broadcast., vol. 66, no. 1, pp. 9-20, Mar. 2020.

[37] H. Yamamoto et al., "A Study on LDM-BST-OFDM transmission for the next-generation terrestrial broadcasting," IEEE Trans. Broadcast. vol. 66, no. 2, pp. 205-215, Jun. 2020.

[38] L. Zhang et al., "Using layered division multiplexing for wireless inband distribution links in next generation broadcast systems," accepted for publication in IEEE Trans. Broadcast.

[39] J. Montalban et al., "Improved semi-blind channel estimation with time domain cancellation for LDM-LSI," IEEE Trans. Broadcast., vol. 66 no. 2, pp. 613-619, Sep. 2020.

[40] D. Gómez-Barquero and O. Simeone, "LDM versus FDM/TDM for unequal error protection in terrestrial broadcasting systems: An information theoretic view," IEEE Trans. Broadcast., vol. 61, no. 4, pp. 571-579, Dec. 2015

[41] T. M. Cover and J. A. Thomas, Element of Information Theory, New York, NY, USA: Wiley, 2006.
[42] S. Ahn et al., "Fronthaul compression and precoding optimization for NOMA-based joint transmission of broadcast and unicast services in CRAN," accepted for publication in IEEE Trans. Broadcast.

[43] S.-I. Park et al., "Transmitter identification signal analyzer for single frequency network," IEEE Trans. Broadcast., vol. 54, no. 3, pp. 383 393, Sep. 2008.

[44] S.-I. Park et al., "An efficient receiver structure for robust data transmission using TxID signal in the ATSC DTV system," IEEE Trans. Consum. Electron., vol. 56, no. 2, pp. 408-414, May 2010.

[45] Y.-W. Suh et al., "A novel TxID insertion system for ATSC DTV auxiliary data transmission," IEEE Consum. Electron., vol. 57, no. 1, pp. 35-39, Feb. 2011

[46] S.-I. Park et al., "RF watermark backward compatibility tests for the ATSC terrestrial DTV receivers," IEEE Trans. Broadcast., vol. 57, no. 2, pp. 246-252, Jun. 2011.

[47] B. Rong et al., "Signal cancellation techniques for RF watermark detection in ATSC mobile DTV system," IEEE Trans. Veh. Technol. vol. 60 , no. 8 , pp. 4070-4076, Oct. 2011.

[48] S.-I. Park et al., "ATSC 3.0 transmitter identification signals and applications," IEEE Trans. Broadcast., vol. 63, no. 1, pp. 240-249, Mar 2017.

[49] J. Lee et al., "Efficient ATSC 3.0 TxID signal detection for single frequency networks," IEEE Trans. Broadcast., vol. 65, no. 2, pp. 326 332, Jun. 2019

[50] S. Ahn et al., "Probabilistic analysis on RF-watermark TxID detection in SFN with randomly distributed co-channel interferers and preamble cancellation," IEEE Access, vol. 8, pp. 56300-56311, Mar. 2020.

[51] S. Kwon et al., "Detection schemes for ATSC 3.0 transmitter identification in single frequency network," IEEE Trans. Broadcast, vol. 66, no. 2, pp. 229-240, Jun. 2020.

[52] J. Lee et al., "Transmitter identification signal detection algorithm for ATSC 3.0 single frequency networks," IEEE Trans. Broadcast, vol. 66 no. 3, pp. 737-743, Sep. 2020.

[53] S. Jeon et al., "Field trial results for ATSC 3.0 TxID transmission and detection in single frequency network of Seoul, in Proc. IEEE BMSB, Valencia, Spain, Jun. 2018

[54] H. Jung et al., "ATSC 3.0 channel bonding performance with equal PLP rate in fixed channel environment," in Proc. IEEE BMSB Valencia, Spain, Jun. 2018

[55] H. Jung et al., "ATSC 3.0 Channel bonding performance in mobile channel environments," in Proc. IEEE BMSB, Jeju Island, South Korea, Jun. 2019.

[56] H. Jung et al., "Implementation of terrestrial $8 \mathrm{~K}$ broadcast system using ATSC 3.0 channel bonding," in Proc. IEEE BMSB, Paris, France, Oct. 2020.

[57] D. Gómez-Barquero et al., "MIMO for ATSC 3.0," IEEE Trans. Broadcast., vol. 62, no. 1, pp. 298-305, Mar. 2016.

[58] S. Saito et al., "8K Terrestrial Transmission field tests using dualpolarized MIMO and higher-order modulation OFDM," IEEE Trans.Broadcast., vol. 62, no. 1, pp. 306-315, Mar. 2016.

[59] H.-J. Yim et al., "Implementation of targeted advertisement services on ATSC 3.0 runtime environment," IEEE BMSB, Jeju Island, South Korea, Jun. 2019.

[60] H.-J. Yim et al., "Application-based targeted advertisement system for ATSC 3.0 UHD service," accepted for publication in IEEE Trans. Broadcast.

[61] J. Lee et al., "Efficient transmission of multiple broadcasting services using LDM and SHVC," IEEE Trans. Broadcast., vol. 64, no. 2, pp. 177-178, Jun. 2018.

[62] S. Ahn et al., "Large-scale network analysis on NOMA-aided broadcast/unicast joint transmission scenarios considering content probability," accepted for publication in IEEE Trans. Broadcast.

[63] D. Vargas and Y. J. D. Kim, "Two-layered superposition of broadcast/multicast and unicast signals in multiuser OFDMA systems,' IEEE Trans. Wireless Commun., vol. 19, no. 2, pp. 979-994, Feb. 2020

[64] J. Zhao et al., "Non-orthogonal unicast and broadcast transmission via joint beamforming and LDM in cellular networks," IEEE Trans. Broadcast., vol. 66, no. 2, pp. 216-228, Jun. 2020

[65] L. Zhang et al., "Using non-orthogonal multiplexing in 5G-MBMS to achieve broadband-broadcast convergence with high spectral efficiency," IEEE Trans. Broadcast., vol. 66, no. 2, pp. 490-502, Jun. 2020

[66] E. Iradier et al., "Using NOMA for enabling broadcast/unicast convergence in 5G networks," IEEE Trans. Broadcast., vol. 66, no. 2, pp. 503-514, Jun. 2020.

[67] L. Zhang et al., "Using layered division multiplexing for wireless inband distribution links in next generation broadcast systems," accepted for publication in IEEE Trans. Broadcast. 
[68] S.-I. Park et al., "Augmented data transmission based on low density parity check code for the ATSC terrestrial DTV system," IEEE Trans. Broadcast., vol. 58, no. 4, pp. 637-641, Dec. 2012.

[69] S.-I. Park et al., "A newly designed quarter-rate QC-LDPC code for the cloud transmission system," IEEE Trans. Broadcast., vol. 59, no.1, pp. 155-159, Mar. 2013.

[70] S.-I. Park et al., "Raptor-like rate compatible LDPC codes and their puncturing performance for the cloud transmission system," IEEE Trans. Broadcast., vol. 60, no.2, pp. 239-245, Jun. 2014

[71] K.-J. Kim et al., "Low-density parity-check codes for ATSC 3.0," IEEE Trans. Broadcast., vol. 62, no.1, pp. 189-196, Mar. 2016.

[72] N.-S. Loghin et al., "Non-uniform constellations for ATSC 3.0," IEEE Trans. Broadcast., vol. 62, no.1, pp. 197-203, Mar. 2016.

[73] H. Jeong et al., "Flexible and robust transmission for physical layer signaling of ATSC 3.0," IEEE Trans. Broadcast., vol. 62, no.1, pp. 204-215, Mar. 2016

[74] J. Barrueco et al., "Constellation design for bit-interleaved coded modulation (BICM) systems in advanced broadcast standards," IEEE Trans. Broadcast., vol. 63, no.4, pp. 603-614, Dec. 2017.

[75] S.-K. Ahn et al., "Comparison of low-density parity-check codes in ATSC 3.0 and 5G Standards," IEEE Trans. Broadcast., vol. 65, no.3, pp. 489-495, Sep. 2019

[76] 3GPP TR 38.913 V15.0.0, "Study on scenarios and requirements for next generation access technologies (Release 15)", Jun. 2018

[77] 3GPP TS 38.212 V15.7.0, "Multiplexing and channel coding (release 15)," Sep. 2019.

[78] 3GPP TR 36.976 V1.0.0, "Overall description of LTE-based 5G broadcast (Release 16)", Dec. 2019

[79] S. -I. Park et al. "Field test results of layered division multiplexing for the next generation DTV system," IEEE Trans. Broadcast., vol.63, no. 1, pp. 260-266, Mar. 2017

[80] ETSI TS 102 831: "Digital Video Broadcasting (DVB); Implementation guidelines for a second generation digital terrestrial television broadcasting system (DVB-T2)," V1.2.1, Aug. 2012.

[81] S. -I. Park et al., "Field comparison tests of LDM and TDM in ATSC 3.0," IEEE Trans. Broadcast., vol.64, no. 3, pp. 637-647, Sep. 2018.

[82] B.-m. Lim et al., "Mobile field comparison test of LDM and TDM based on ATSC 3.0," in Proc. IEEE BMSB, Cagliari, Italy, Jun. 2017.

[83] B.-m. Lim et al., "Mobile Testing of ATSC 3.0 MISO in SFN," in Proc. IEEE BMSB, Jeju Island, South Korea, Jun. 2019.

[84] S. Ahn et al., "Multi-antenna diversity gain in terrestrial broadcasting receivers on vehicles: A coverage probability perspective," submitted to ETRI J.

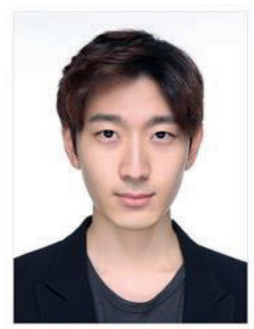

Sungjun Ahn received B.S. and M.S. degree in electrical engineering from Korea Advanced Institute of Science and Technology (KAIST), Daejeon, South Korea, in 2015 and 2017, respectively. $\mathrm{He}$ has been with Media Research Division, Electronics and Telecommunications Research Institute (ETRI) since 2017, where he is currently a Research Engineer. His research interests include stochastic geometry analysis, signal processing, and optimization for wireless communications and digital broadcasting.

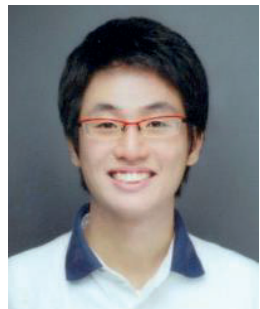

Sunhyoung Kwon received the B.S degree in electrical communications engineering from Information and Communications University, Daejeon, Korea, in 2008, and the M.S. and Ph.D. degree in electrical engineering from the Korea Advanced Institute of Science and Technology, Daejeon, in 2010 and 2020, respectively. Since 2010, he has been with the Media Research Division, Electronics and Telecommunication Research Institute, where he is currently a senior member of research staff. His research interests are in the area of digital broadcasting and communications.

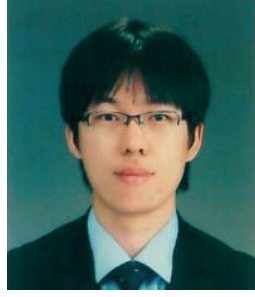

Seok-Ki Ahn received the B.S., M.S., and $\mathrm{Ph} . \mathrm{D}$. degrees in electronics and electrical engineering from Pohang University of Science and Technology (POSTECH) in 2006, 2008, and 2013, respectively. From 2010 to 2013, he was a student on scholarship at the Digital Media and Communications R\&D Center, Samsung Electronics. From 2013 to 2018, he was a Senior Engineer with Samsung Electronics, Suwon, South Korea. He is currently with Electronics and Telecommunications Research Institute (ETRI), Daejeon, South Korea. His research interests include channel coding, MIMO transceiver design, and broadband communications.

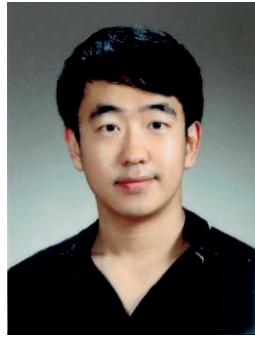

Hoiyoon Jung received the B.S. and M.S. degree in information and communication engineering from Korea Advanced Institute of Science and Technology (KAIST), Daejeon, South Korea, in 2006 and 2008, respectively. $\mathrm{He}$ has been with Media Research Division, Electronics and Telecommunications Research Institute (ETRI) since 2008, where he is currently a Senior Researcher. His research interests include $5 \mathrm{G}$ cellular system and next generation terrestrial broadcasting system.

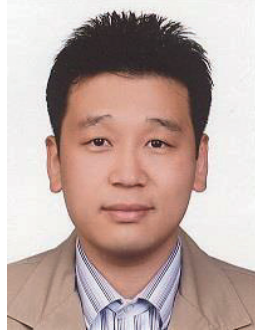

Sung-Ik Park received the B.S.E.E. degree from Hanyang University, Seoul, South Korea, in 2000 and the M.S.E.E. degree from POSTECH, Pohang, South Korea, in 2002, and the Ph.D. degree from Chungnam National University, Daejeon, South Korea, in 2011. Since 2002, he has been with the Media Research Division, Electronics and Telecommunication Research Institute (ETRI), where he is Project Leader and Principal Member of Research Staff. His research interests are in the area of error correction codes and digital communications, in particular, signal processing for digital television. He has over 200 peer-reviewed journal and conference publications, and multiple best paper and contribution awards for his work on broadcasting technologies. He currently serves as an Associate Editor for the IEEE TRANSACTIONS ON BROADCASTING and ETRI Journal, and a Distinguished Lecturer of IEEE Broadcasting Technology Society. 